\title{
The effectiveness of a chart audit and feedback intervention to improve cardiology provider delivery of heart failure self-care education
}

Dorothy Murphy

Follow this and additional works at: https://researchrepository.wvu.edu/etd

\section{Recommended Citation}

Murphy, Dorothy, "The effectiveness of a chart audit and feedback intervention to improve cardiology provider delivery of heart failure self-care education" (2015). Graduate Theses, Dissertations, and Problem Reports. 6283.

https://researchrepository.wvu.edu/etd/6283

This Dissertation is protected by copyright and/or related rights. It has been brought to you by the The Research Repository @ WVU with permission from the rights-holder(s). You are free to use this Dissertation in any way that is permitted by the copyright and related rights legislation that applies to your use. For other uses you must obtain permission from the rights-holder(s) directly, unless additional rights are indicated by a Creative Commons license in the record and/ or on the work itself. This Dissertation has been accepted for inclusion in WVU Graduate Theses, Dissertations, and Problem Reports collection by an authorized administrator of The Research Repository @ WVU.

For more information, please contact researchrepository@mail.wvu.edu. 
The effectiveness of a chart audit and feedback intervention to improve cardiology provider delivery of heart failure self-care education

\author{
Dorothy Murphy, MS, FNP-BC \\ DNP Capstone submitted to the School of Nursing at \\ West Virginia University \\ In partial fulfillment of the requirements for the degree of \\ Doctor of Nursing Practice
}

Jennifer Mallow, Ph.D., Chair

Emily Barnes, D.N.P.

Dana Woody, M.S.

Morgantown, West Virginia

2015

Keywords: [chart audit, provider feedback, performance improvement, heart failure, selfcare education]

Copyright 2015 [Dorothy L. Murphy] 


\begin{abstract}
Heart failure is a challenging disease process that presents a high risk of mortality, frequent hospitalizations, and an overwhelming economic burden to the health system. Patient self-care education has been shown to decrease unplanned hospitalizations, emergency room visits, hospitalized days, costs, and mortality rates. However, only 61\% of adults with HF receive self-care education within a 12-month period.

Under the framework of the Iowa Model for Evidence-Based Practice, the purpose of this project was to implement an electronic health record audit and written and verbal provider feedback intervention with the aim of improving provider delivery of heart failure self-care education to adults with heart failure with reduced ejection fraction. The objectives of this project were to: 1 ) Improve cardiology provider performance of heart failure self-care education delivery to adult patients with heart failure and 2) Cardiology providers will report satisfaction with the chart audit and performance feedback process.
\end{abstract}

Data analysis revealed an improvement of provider delivery of heart failure selfcare education in all heart failure self-care education elements. This project provided the opportunity to design, develop, and evaluate an evidence-based initiative aimed at improving health care quality. Furthermore, this project has provided a foundation for future clinical initiatives aimed at improving provider delivery of heart failure self-care education. 


\section{Acknowledgements}

Throughout the course of this project I have received support and encouragement from a great number of individuals. Dr. Jennifer Mallow has been a mentor and colleague. Her guidance as Committee Chair has made this project a thoughtful and rewarding journey. I would like to thank my Capstone Committee of Dr. Emily Barnes and Mrs. Dana Woody for their expertise and support. I would also like to thank Dr. Chad Hoyt, Dr. Steve Rennyson and The Stroobants’ Cardiovascular Center for their gracious support of this project. Finally, I would like to acknowledge my family for their sacrifice and support they have provided over the course of completing this program. 


\section{Table of Contents}

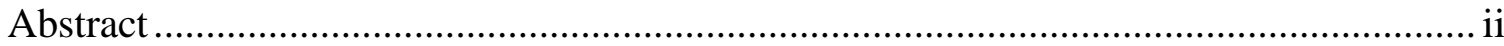

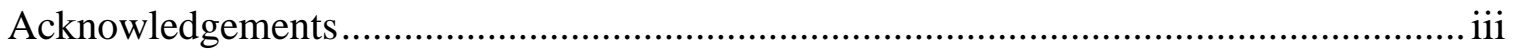

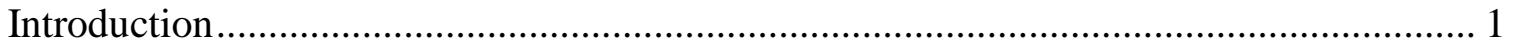

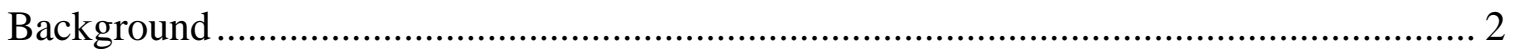

Burden of Heart Failure .................................................................................................................

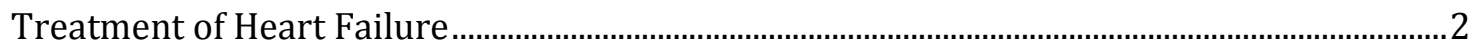

Heart failure self-care (HFSC) education. .................................................................................

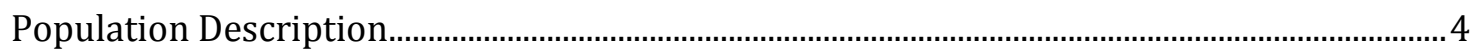

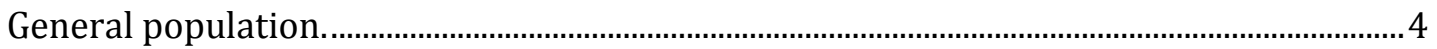

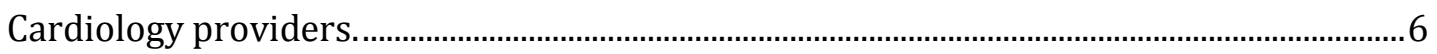

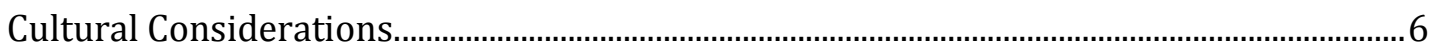

Statement of the Problem.....................................................................................................................

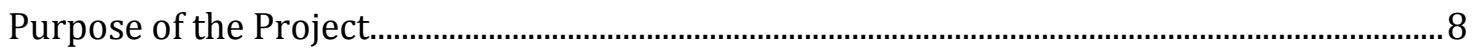

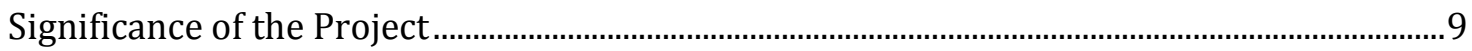

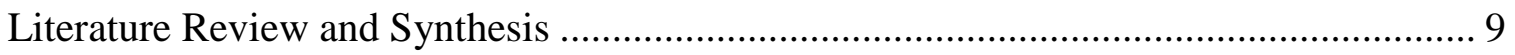

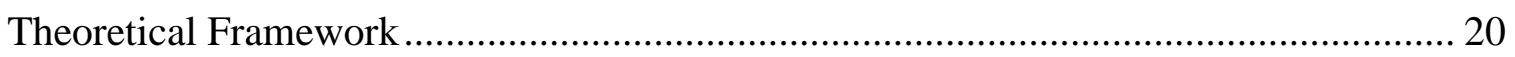

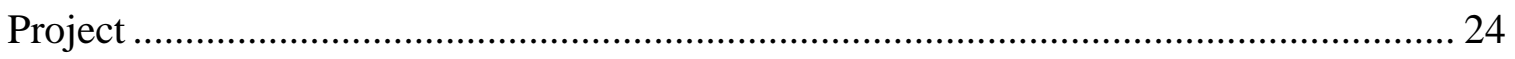

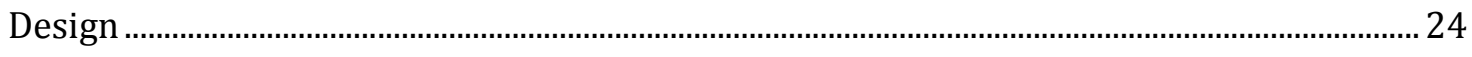

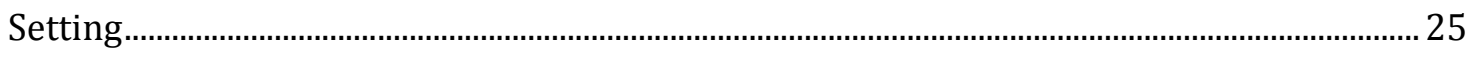

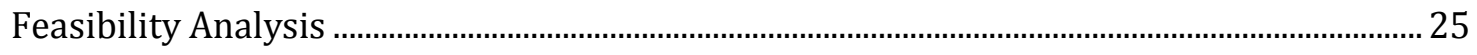

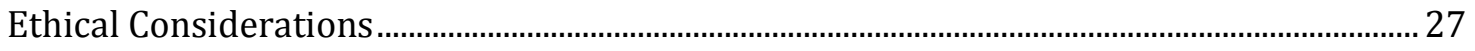

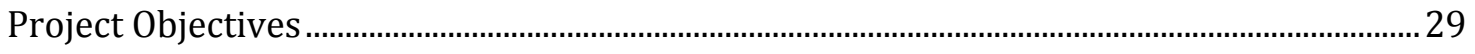

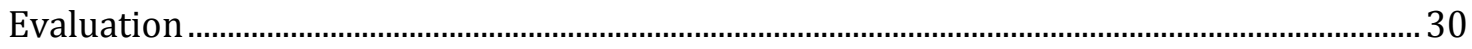

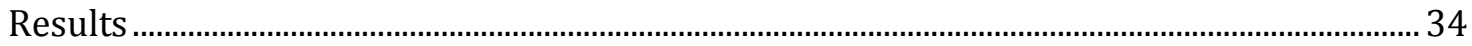

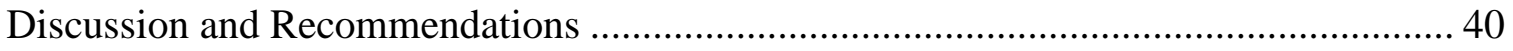

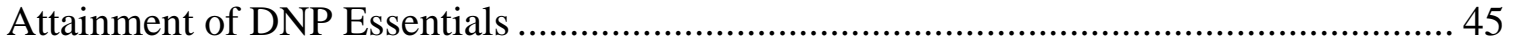

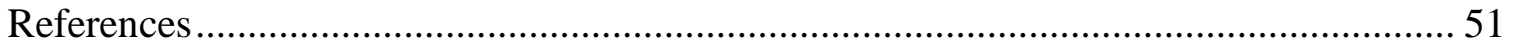

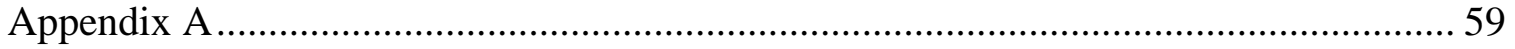

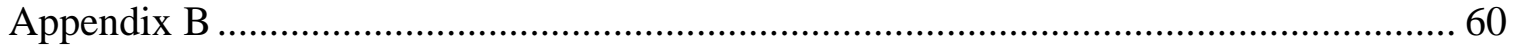

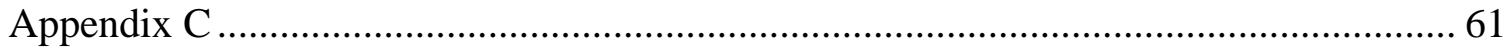

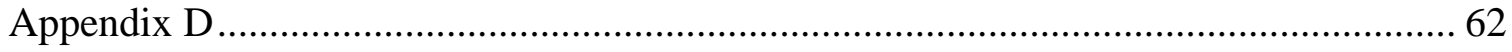

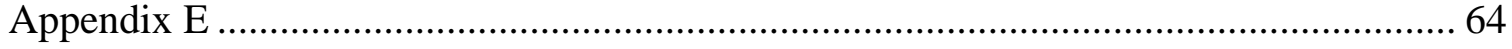

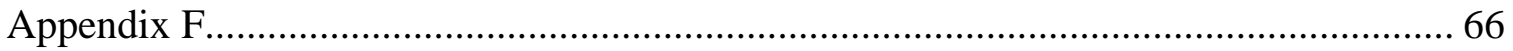


IMPROVE HF SELF-CARE EDUCATION DELIVERY

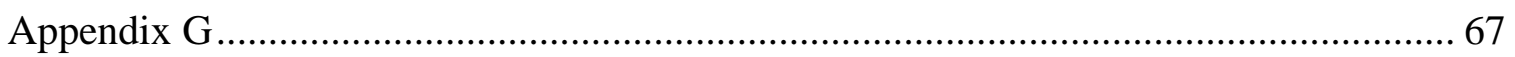

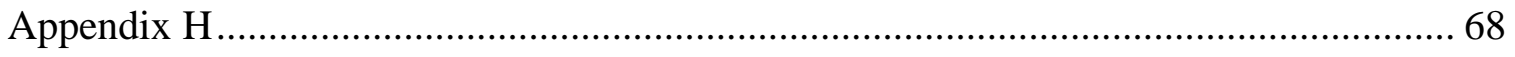

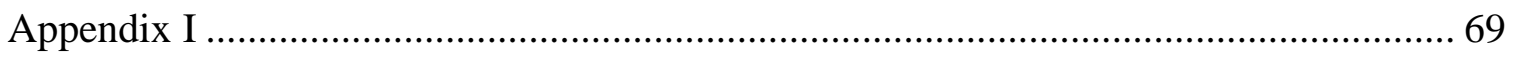

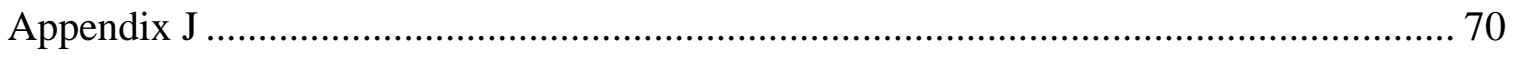




\section{IMPROVE HF SELF-CARE EDUCATION DELIVERY}

\section{Introduction}

Heart failure with reduced ejection fraction (HFrEF) is a multifactorial, complex, and deleterious syndrome. Heart failure causes progressive clinical syndrome of fluid overload that causes symptoms of dyspnea, fatigue, edema, hemodynamic compromise, and ultimately death (Yancy et al., 2013). Heart failure decreases an individual's quality of life while causing an increase in morbidity, mortality, and resource utilization (Yancy et al., 2013). The desired outcomes of HF management include the reduction of death, hospitalization, future clinical deterioration, and symptoms of heart failure and improvement of patient's activity level, ability to manage their own illness, and overall sense of well-being and health status (Yancy et al., 2013). Despite significant therapeutic advancements over the past several decades aimed at reducing the burden of HF, health outcomes remain suboptimal (Go et al., 2013; Fonarow et al., 2010).

Current practice guidelines recommend that providers deliver self-care education to individuals with HF (Lindenfeld et al., 2010; Yancy et al., 2013). Patient self-care education has been shown to decrease unplanned hospitalizations, emergency room visits, hospitalized days, costs, and mortality rates (Boren, Wakefield, Gunlock, \& Wakefield, 2009). According to data from the Registry to Improve the Use of Evidence-Based Heart Failure Therapies in the Outpatient Setting (IMPROVE HF), only 61\% of adults with HF receive self-care education within a 12-month period (Fonarow et al., 2010). Self-care education is a complex intervention in which health care providers face challenges delivering education and patients experience challenges receiving education. Chart audit and performance feedback is an intervention that has demonstrated improvements in provider delivery of evidence-based care (Ivers et al., 2013; Jamtvedt, Young, 
IMPROVE HF SELF-CARE EDUCATION DELIVERY

Kristoffersen, OBrian, \& Oxman, 2006; Okelo et al., 2014). The purpose of this project was to implement an electronic health record (EHR) audit and provider feedback intervention with the aim of improving provider delivery of HF self-care (HFSC) education to adults with HFrEF.

\section{Background}

\section{Burden of Heart Failure}

Heart failure is a challenging disease process that presents a high risk of mortality, frequent hospitalizations, and an overwhelming economic burden to the health system. It is estimated in the United States that over 5 million adults have HF and it is projected that from 2013 to 2030 HF prevalence will increase by 25\% (Go et al., 2013). Approximately 650,000 new cases of HF are diagnosed annually in adults’ 45-years and older (Go et al.). In the U.S., HF is one of the most frequent causes of hospitalization in individuals 65years and older, accounting for more than $70 \%$ of the estimated 1 million hospitalizations in 2010 (CDC, 2010). The economic cost in the U.S. exceeds $\$ 40$ billion annually and is projected to increase to $\$ 70$ billion by 2030 (Go et al.; Roger et al., 2012).

\section{Treatment of Heart Failure}

Over the past half-century, significant advancements have been made in the management of HF. Effective HF treatment requires both pharmacologic and nonpharmacological management by health provider and self-care management by the patient. Pharmacological and device therapies have significantly reduced morbidity, mortality, and health-associated cost and significantly improved patient's quality of life (Yancy et al., 2013). Heart failure self-care is complex. Patients with HF are challenged daily to understand the complex disease process, therapeutic management and risk 
IMPROVE HF SELF-CARE EDUCATION DELIVERY

modification strategies, and the ability to link signs and symptoms that require a welldefined action plan. Ultimately, the corner stone of successful HF management is the patient's ability to perform self-care behaviors (Boren et al., 2009; Riegal et al., 2004). Effective self-care among individuals with HF is associated with reduced rehospitalization and mortality rates (Lee, Moser, Lennie, Riegel, 2011; McDonald, 2010; Whellan, Hasselblad, Peterson, O’Connor, \& Schulman, 2005). Furthermore, failed selfcare is linked to HF exacerbation, re-hospitalization, and increased cost of care (Lee, Chavez, Baker, \& Luce, 2004; Liao et al., 2006; Liao et al., 2007; Linne, Liedhol, Jendteg, \& Israelsson, 2000)

Heart failure self-care (HFSC) education. Provider-delivered HFSC education is an American College of Cardiology Fellowship/American Heart Association (ACCF/AHA) Class I (Level of Evidence B) recommendation (Yancy et al., 2013). The Heart Failure Society of America (HFSA) has identified and recommended essential elements that constitute HFSC education. Consistent with HFSA (2010) recommendations, HFSC education is comprised of the following elements:

1) Definition and discussion of heart failure including the patient's cause of heart failure, symptoms, and treatments (Essential 1).

2) Identifying and discussing specific signs and symptoms of escalating symptoms and a well-defined action for responding to certain symptoms (Essential 2).

3) Reiterate the indication and use of medications (Essential 3)

4) Reiterate the necessity to modify risk factors associated with HF progression (Element 4).

5) Defining and reiterating dietary recommendations (Essential 5). 
IMPROVE HF SELF-CARE EDUCATION DELIVERY

6) Prescribing individual plan for physical activity (Element 6).

7) Discuss the importance of treatment adherence and well-defined strategies to promote adherence (Element 7).

Factors affecting heart failure self-care education. Self-care education has been demonstrated to improve patient's knowledge of HF, self-monitoring, medication adherence, and to reduce hospital days (Boren et al., 2009). When implemented effectively, patient teaching is individualized, encourages patient autonomy and self-care, and is integrated into every patient encounter by every member of the health care team (Lindenfeld et al., 2010; Falvo, 2011; Porche, 2007). Health care providers face many challenges to delivering effective patient education. Barriers that providers face in patient education delivery relate to individual patient factors, time constraints, and organizational support (Lindenfeld et al., 2010). Patients encounter challenges to performing HFSC including limited health literacy, motivation, and self-efficacy, adjustment to health and life-related stresses, social support, and environmental factors (Falvo, 2011).

\section{Population Description}

General population. This project was conducted at a private cardiology practice in Lynchburg, Virginia. According to the United States Census Bureau (2012), 63.4\% of the people residing in the city of Lynchburg are Caucasian and 14\% are 65-years and older compared with 13\% in the Commonwealth of Virginia and 13.7\% in the U.S. Eighty-five percent are high school graduates and 30.1\% have a bachelor's degree or higher (U.S. Census). Poverty is strikingly higher in the city of Lynchburg at $22.1 \%$

(U.S. Census, 2012) compared to $10.7 \%$ in the Commonwealth of Virginia and $14.3 \%$ in 
IMPROVE HF SELF-CARE EDUCATION DELIVERY

the U.S. (U.S. Census, 2012). The homeownership rate is $55.4 \%$ and the median household income is $\$ 37,733$ annually, which is only $60 \%$ and $70 \%$ of the median household income of the Commonwealth of Virginia and the U.S., respectively (U.S. Census). The unemployment rate in the city of Lynchburg is $8.2 \%$ compared to $6.2 \%$ in the Commonwealth of Virginia (U.S. Census). Social factors have been linked to negative heart failure outcomes. Specifically, older age and low socioeconomic status are associated with HF hospital readmissions (Calvillo-King et al., 2013). The present social factors in the project setting, including higher rates of poverty, lower median household income, and higher unemployment enforces the importance of interventions aimed at improving HF outcomes.

Risky health behaviors have been associated with higher HF hospital readmission rates (Calvillo-King et al., 2013). In general, the city of Lynchburg has a higher rate of risky health behaviors than the Commonwealth of Virginia (U.S. Census, 2012). Additionally, the availability of healthy nutrition and recreation is limited (The Robert Wood Johnson Foundation, 2012). According to the Robert Wood Johnson Foundation (2012), rates of poor health (15\%), adult obesity (31\%), adult smoking (22\%), and physical inactivity (30\%) are higher in Lynchburg, VA than the state's average of poor health (14\%), adult obesity (28\%), adult smoking (18\%), and physical inactivity (24\%). The higher rates of risky health behaviors, specifically smoking, enforces the importance of this project that aims to improve delivery of HFSC education that includes patient risk factor reduction education.

According to the Center for Disease Control and Prevention (2010), the HF hospitalization rate per 1,000 Medicare beneficiaries, 65-years and older is $16.4 \%$ in the 


\section{IMPROVE HF SELF-CARE EDUCATION DELIVERY}

city of Lynchburg, Virginia. Heart failure mortality for all races and gender in individuals 65-years and older is higher in Lynchburg (601 per 100,000) compared to the rest of Virginia (579.9 per 100,000) (CDC, 2010). The HF hospitalization rate among Medicare beneficiaries in Lynchburg, Virginia (16.4 per 1,000) is essentially equal to the Commonwealth of Virginia (16.1 per 1,000) and National (16.8 per 1,000) rates (CDC, 2010).

Cardiology providers. This project was conducted at a private cardiology specialty practice in Lynchburg, Virginia. The target population for this project was cardiology providers who deliver care for adults with HFrEF in the Lynchburg Office. For the purposes of this project, healthcare providers who practice in a specialty cardiology clinic, including physicians, nurse practitioners, and physician assistants were included. There are over 600 physicians, nurse practitioners, and physician assistants in the greater Lynchburg region. There are 29 cardiologists in the greater Lynchburg region, 22 of which practice at the cardiology specialty site this capstone project was conducted at (Stroobants’ Cardiology, 2014). There are more than 50 advanced practice nurses in the greater Lynchburg region, 8 of which practice at the cardiology specialty site that this project was conducted at (Stroobants' Cardiology, 2014). There are over 40 physician assistants in the greater Lynchburg region, 3 of which practice at the cardiology specialty site that the project was conducted at (Stroobants’ Cardiology, 2014).

Cultural Considerations. Geographic region, heritage, and culture affect the health and health behaviors of a population. Lynchburg is located just beyond the southeastern margin of the Appalachian Region (Appalachian Region, 2008). While the City of Lynchburg is marginally outside of the geographic region of Appalachia, the 


\section{IMPROVE HF SELF-CARE EDUCATION DELIVERY}

populations served from neighboring counties are geographically situated in Appalachia.

In the Appalachian culture, individuals are known for a strong faith in God and determination to be self-reliant (Huttlinger \& Purnell, 2008). In Appalachia, health is described as optimal functioning of the body, mind, and spirit (Huttlinger \& Purnell). Disease is a part of life and not a priority of concern unless it interferes with personal or family functioning. Therefore, the emphasis on health promotion and disease prevention has not been fully embraced by the Appalachian population (Huttlinger \& Purnell).

Appalachians typically embody a strong sense of self-reliance though health outcomes are ultimately viewed in God's control (Huttlinger \& Purnell, 2008). A strong sense of self-reliance may provide opportunity for health self-management and improved health outcomes (Huttlinger \& Purnell). Paradoxically, Appalachians tend to feel powerless in the healthcare system and are inclined to let go of their own care and place high expectations that their healthcare provider is fully responsible for their healthcare (Huttlinger \& Purnell). While a dependent attitude may be reasonable in the acute care setting, it may be a barrier to the patient's day-to-day management of heart failure.

Communities in Appalachia have historically lacked health care providers, particularly specialty care providers. Appalachian self-reliance manifests as a strong trust in their own people and a mistrust of outsiders (Huttlinger \& Purnell, 2008). Historic lack of healthcare providers native to the Appalachian culture along with a culture of selfreliance may provide insight as to why health care, despite being readily available, is underutilized in Appalachia (Huttlinger, Schaller-Ayers, \& Lawson, 2004). Healthcare providers must gradually earn the trust of the community based upon their personal characteristics and behavior, more so than knowledge (Huttlinger \& Purnell, 2008). 
IMPROVE HF SELF-CARE EDUCATION DELIVERY

Providers who are not indigenous to the Appalachian region may face a cultural barrier to delivering self-care education. Cardiology specialty physicians are more likely to be

nonnative to the Appalachia region and culture compared to nurses (Huttlinger \& Purnell, 2008). Therefore, the native cardiology providers, particularly nurses, may be better positioned to provide culturally-relevant self-care education.

\section{Statement of the Problem}

Patient self-care education is an ACCF/AHA Class I, Level of Evidence B recommendation. Self-care education has been demonstrated to improve patient knowledge, self-monitoring, and medication adherence (Yancy et al., 2013).

Furthermore, patient self-care educational interventions have been shown to decrease unplanned hospitalizations, emergency room visits, hospitalized days, costs, and mortality rates (Boren et al., 2009). Despite the consequences of high costs, poor quality of life and increased morbidity and mortality related to inadequately managed HF, and while there is strong evidence supporting the effectiveness of self-care education in the management of HF, nearly $40 \%$ of patients with HF do not receive self-care education from cardiology providers (Fonarow et al., 2010).

\section{Purpose of the Project}

The purpose of this capstone project was to implement and evaluate a chart audit and provider performance feedback intervention aimed at improving provider delivery of HFSC education. Audit and feedback interventions have demonstrated improvement in provider adherence to standards of care (Ivers et al., 2013). More specifically, the chart audit and provider performance feedback intervention has been demonstrated to improve provider delivery of self-care education (Okelo et al., 2014). 
IMPROVE HF SELF-CARE EDUCATION DELIVERY

\section{Significance of the Project}

In the United States, it is estimated that HF affects 5.1 million adults (Go et al., 2013). At the time of diagnosis, an individual's 5-year survival rate is approximately 50\% (Levy et al., 2002; Roger et al., 2004). Heart failure is the primary diagnosis at discharge for over 1 million hospitalizations annually (National Heart, Lung, and Blood Institute, 2006). Heart failure is estimated to cost the U.S. \$32 billion annually and the cost is projected to increase by $120 \%$ by 2030 to $\$ 70$ billion (Heidenreich et al., 2011). Given the high prevalence, mortality, morbidity, and economic burden associated with HF, evidence-based practice is a high priority. Self-care education is a guideline-driven, well-substantiated strategy to improve health outcomes of individuals with HF (Lindenfeld et al., 2010: Yancy et al., 2013). Despite the evidence supporting delivery of HFSC education, less than $40 \%$ of individuals with HF receive education during a $12-$ month period (Fonarow et al., 2010). Audit and feedback interventions have demonstrated improvement in provider performance, specifically related to delivery of self-care education (Ivers et al., Okelo et al., 2014). The purpose of this capstone project was to implement audit and performance feedback aimed to improve cardiology provider HFSC education delivery. This capstone was proposed to evaluate outcomes, verify provider performance and provide opportunity for exploring further practice or system solutions for improving HFSC education delivery.

\section{Search Strategy}

\section{Literature Review and Synthesis}

The search strategy to identify the best evidence related to the use of audit and feedback intervention aimed at improving provider behavior and included a search of the Cochrane Library, Ovid, EMBASE, CINAHL, and PUBMED. Keywords and phrases 
IMPROVE HF SELF-CARE EDUCATION DELIVERY

used for the search included audit, feedback, chart review, provider performance and provider documentation. No limits were placed on type of article or publication date. The search was limited to the English language and available full text for convenience. These searches resulted in 615 articles found.

\section{Study Identification}

The search was narrowed by publication date (2009-2014), study design (systematic review or controlled clinical trial) and available full text, which yielded 74 studies for review. Abstracts of 74 studies were reviewed for selection criteria. If any of the selection criteria were not indicated as a primary component, then the study was excluded from this review. If the abstract did not provide adequate information to determine selection criteria, the full text publication was retrieved and reviewed. Studies that were included in identified systematic reviews (Ivers et al., 2013; Okelo et al., 2014) were not individually appraised or discussed in this review. A search of the National Guidelines Clearinghouse was conducted for professional guidelines, pertaining to heart failure disease management and quality improvement, were reviewed to inform this project. This search led to the identification of two clinical practice guidelines (Yancy et al., 2013; Lindenfeld et al., 2010). Thorough review of the ACCF/AHA 2013 Guideline for the Management of Heart Failure resulted in discovering one prospective controlled trial (Fonarow et al., 2010). Finally, one strong systematic review (Ivers et al., 2013), one moderate-strength systematic review (Okelo et al., 2014), two clinical practice guidelines (Yancy et al., 2013; Lindenfeld et al., 2010), and one prospective trial (Fonarow et al., 2010) were included in this review.

\section{Selection Criteria}


IMPROVE HF SELF-CARE EDUCATION DELIVERY

Population. The focus of this review was the health care provider, defined here as physicians, advanced practice nurses, and physician assistants.

Intervention. The intervention of interest was chart audit and provider performance feedback.

Outcomes. The principle outcome of interest was improvement in provider behavior in adhering to a standard of care or practice guideline.

Study Design. Articles were included if classified as a systematic review, clinical practice guideline, or controlled trial.

\section{Methods for Quality Assessment}

A single reviewer critically appraised the quality of each manuscript. Clinical practice guidelines were appraised according to the Appraisal of Guidelines Research \& Evaluation (AGREE, 2006). The systematic reviews and clinical trial were appraised according the Scottish Intercollegiate Guideline Network (SIGN, 2008). Each manuscript was assigned a LOE according to Larrabee’s (2009) system of hierarchy. Quality assessment is depicted in Appendix A.

\section{Systematic Reviews}

A strong systematic review of randomized controlled trials (RCTs) by Ivers, et al. (2012), evaluated audit and feedback intervention effects on the practice behaviors of healthcare professionals at the post- graduate level or higher. The purpose of this systematic review was to assess the effects of audit and feedback on the practice of healthcare professionals and patient outcomes. Furthermore, Ivers et al. (2012) examined factors that may explain variation in the effectiveness of an audit and feedback intervention. 
IMPROVE HF SELF-CARE EDUCATION DELIVERY

The systematic review included 140 RCTs in which an audit and feedback intervention was the core intervention delivered to post-graduate health care professionals. Ivers et al., (2012) conducted multivariate meta-regression to evaluate factors that may explain the variation in the effectiveness of interventions across RCTs. Factors included in the meta-regression included baseline performance, profession of recipients, source of feedback, format of feedback, frequency of feedback, instructions for improvement, direction of change required, and risk of bias within the RCT.

Outcomes were categorized as dichotomous (provider behavior and health outcomes) or continuous (number of laboratory or prescription orders). Ivers et al. (2012) reported that RCTs featuring dichotomous outcomes across 49 RCTs demonstrated a weighted mean adjusted risk difference (RD) was $4.3 \%$ with an interquartile range (IQR) $0.5 \%$ to $16 \%$ absolute increase in health care professionals’ compliance with desired practice. Analysis of continuous outcomes across 21 RCTs found weighted median adjusted percent change relative to control of 1.3\% (IQR 1.3\% to 28.9\%). Multivariable meta-regression revealed that feedback might be more compelling when baseline performance is low, feedback is provided by a colleague or supervisor, delivered in written and verbal formats on a continuous monthly basis, and incorporates straightforward goals and action plan to achieve the goals. Ivers et al. (2013) substantiated a strong systematic review comprised of a considerable number of RCTs $(n=140)$ and a stable median effect size that support that audit and feedback intervention is effective at improving provider behavior. A potential weakness of this systematic review is that suboptimal documentation may increase the risk of reporting bias. 
IMPROVE HF SELF-CARE EDUCATION DELIVERY

Okelo et al., 2014 conducted a systematic review to determine if interventions targeting health care providers improve adherence to practice guidelines. Of the 86 studies reviewed, 35 were RCTs. There was heterogeneity among the study populations, interventions, and outcomes. Studies were classified based upon intervention and provider audit and feedback was evaluated independently. Specifically, 5 studies (Baker et al., 2003; Feder et al., 1995, Coleman et al., 2003; Richman et al., 2000; Schneider et al., 2000) evaluated provider audit and feedback intervention on provider performance of self-care education. Four studies (Feder et al., 1995, Coleman et al., 2003; Richman \& Poltawsky, 2000; Schneider et al., 2000) demonstrated significant increases (1-40\%) in provider delivery of self-care education. Strengths of this systematic review include the use of well-defined methodology for evaluating the evidence established by the Agency for Healthcare Research and Quality (AHRQ) and tools for minimizing bias from the Cochrane Collaboration. One significant weakness of this systematic review is that over half of the studies were not RCTs $(n=51)$, which is more likely to suggest a beneficial effect of the intervention than randomized trials.

\title{
Clinical Practice Guidelines
}

\section{American College of Cardiology Foundation/American Heart Association.}

\author{
The American College of Cardiology Foundation/American Heart Association
}

(ACCF/AHA) 2013 Guideline for the Management of Heart Failure is an evidence-based practice guideline that serves to assist clinicians in selecting best practices in the detection, management, and prevention of heart failure. Data analysis and development of recommendations are guided by an evidence-based methodology developed by the ACCF/AHA Task Force on Practice Guidelines. Practice recommendations are classified 
IMPROVE HF SELF-CARE EDUCATION DELIVERY

as Class of Recommendation (COR) and Level of Evidence (LOE). The COR describes

the size of the treatment effect and weighs risk, benefit, and if agreement that a particular treatment has been determined useful or may cause harm. Class of recommendation ranges from Class I indicating that a procedure or treatment should be performed to Class III suggesting that a procedure or treatment either has no benefit or may cause harm. The LOE is an evaluation of the strength of the evidence. Level of evidence range from Level A indicating data is derived from multiple RCTs or meta-analyses to Level C indicating recommendation is based upon consensus or expert opinion, case studies, or standard of care.

According to the ACCF/AHA 2013 Guideline for the Management of Heart Failure, two key recommendations are pertinent to this project proposal. Clinicians are recommended to 1) deliver HFSC education (Class I, LOE B Recommendation) and 2) measure and compare quality of care for HF (Class I, LOE B Recommendation). Furthermore, the ACCF/AHA (2013) recommends quality measurement of providerdelivered patient self-care education based upon the IMPROVE HF registry performance measurement criteria. Strengths of this practice guideline include that the guideline is an exhaustive systematic review of evidence pertaining specifically to providers caring for individuals with heart failure, the guideline is informed and written in collaboration with multiple professional societies and the methodology steering the guidelines are evidencebased and well defined. Furthermore, in an effort to minimize bias, the ACCF/AHA members who have industry relationships are not permitted to draft or vote on any recommendation or text that pertains to their industry relationships. One significant weakness of this guideline is that with the exception of two advanced practice nurses, the 
IMPROVE HF SELF-CARE EDUCATION DELIVERY

task force and writing committees are composed of physicians, thus lacking an interdisciplinary team.

Heart Failure Society of America. The Heart Failure Society of America 2010 Comprehensive Heart Failure Practice Guideline is an evidence-based practice guideline that serves to provide recommendations for the management and health care maintenance of individuals with chronic HF. The HFSA utilizes Ovid Medline and PubMed electronic databases to conduct systematic review of the literature to inform practice recommendations.

The quality and strength of the evidence is weighted according to a hierarchical rating scheme. Level A Evidence ratings are reserved for randomized, controlled, and clinical trials. Level B Evidence ratings are assigned to cohort and case control studies, post hoc, subgroup analysis, meta-analysis, prospective observational studies or registries. Level C Evidence ratings are assigned to observational studies, epidemiological findings, and large-scale safety reports. The strength of recommendations is classified as "is recommended”, "should be considered”, “may be considered", or "is not recommended”. Strength of recommendation of "is recommended” is assigned to recommendations that should be implemented as routine care with minimal exceptions. Strength of recommendation of "should be considered: is assigned to interventions that should be implemented for the majority of individuals with some discretion for individualized cases. Strength of "may be considered" is assigned to recommendations where individualization of therapy is indicated, weighing potential benefit and risks. Strength "is not recommended” is assigned to interventions that should not be implemented. 
IMPROVE HF SELF-CARE EDUCATION DELIVERY

The HFSA 2010 Comprehensive Heart Failure Practice Guideline provides detailed, evidence-based management of heart failure including self-care education elements. It is recommended (Strength of Evidence $=\mathrm{B}$ ) that individuals with HF and their caregivers receive individualized education and counseling that emphasizes selfcare. The following educational elements comprise the HFSA (2010) recommendations for HFSC education delivery: 1) definition and cause of HF; 2) recognition of escalating symptoms and plan for response to symptoms; 3) indications for use of each medication; 4) modify risks for HF progression; 5) specific diet recommendations; 6) specific activity/exercise recommendation; and 7) importance of treatment and behavioral strategies to promote treatment adherence.

Strengths of this practice guideline include that the guideline is a clearly defined methodological review of evidence conducted by HF experts written specifically to providers caring for individuals with heart failure. As with the ACCF/AHA 2013 guideline, one significant weakness of the HFSA $2010 \mathrm{HF}$ guideline is that with the exception of two doctoral-prepared nurses, the guideline committee and executive council are composed of physicians and lacks an interdisciplinary team of reviewers. Furthermore, while the guideline provides detailed list of financial interest of the committee, there is no disclaimer of committee members who have financial conflict of being relegated from influencing guideline recommendations.

\section{Clinical Trials}

The Registry to Improve the Use of Evidence-Based Heart Failure Therapies in the Outpatient Setting (IMPROVE HF) registry was a large scale, multi-site, prospective study. The IMPROVE HF registry was designed to evaluate the effectiveness of a 
IMPROVE HF SELF-CARE EDUCATION DELIVERY

performance improvement intervention on cardiology provider use of guideline recommended therapies for individuals with HFrEF (Fonarow et al., 2010). Provider delivered HF self-care education was one of the nine quality measures that were prospectively selected by the committee based upon the HFSA 2010 practice guideline recommendations. Chart review was conducted to determine if written and/or verbal HF education was provided to patients at least 18-years old with a diagnosis of HF. Heart failure self-care education quality measure was met if there was documentation that the patient had received at least 3 of the 7 educational topics over the prior 12-month period. Baseline findings revealed that $61 \%$ of individuals had received recommended HF selfcare education.

The IMPROVE HF registry performance improvement protocol consisted of a multifaceted intervention that included clinical decision support, educational materials, practice-specific data reports, quality measurement benchmarking reports, and structured educational interventions. Twelve and 24-month follow up revealed statistically significant ( $\mathrm{p}<0.001)$ improvements, 69.4 (65.9-73) and 72.1 (68.3-75.9), respectively (Fonarow et al., 2010). Strengths of this prospective study include the substantial setting, population size, and duration of the study. The IMPROVE HF registry was conducted across 167 cardiology outpatient clinics and close to 35,000 patients with HF. The largescale provider-targeted intervention spanned over 24 months. One important limitation of this study as support for this project is that audit and feedback intervention cannot be evaluated independently of the other provider supporting interventions. Additionally, data collection was obtained via chart review. These findings may be limited by inaccurate or incomplete provider documentation. Health care providers need to consider 
IMPROVE HF SELF-CARE EDUCATION DELIVERY

the benefits of providing HF self-care education. Therefore, the merit and validity of the findings, particularly provider-delivered HF self-care education, is contingent upon the accuracy and completeness of provider documentation.

\section{Synthesis}

Evidence from one strong systematic review (Ivers et al., 2013), one moderate strength systematic review (Okela et al., 2010), two strong clinical practice guideline (Lindenfield, et al., 2010; Yancy et al., 2013), and one strong prospective study (Fonarow et al., 2010) supports the use of an audit and feedback intervention aimed at improving provider adherence to practice guidelines recommending delivery of HF self-care education to adults with HF. Heart failure self-care education should include a minimum of 3 of the 7 educational elements supported by the HFSA 2010 Guidelines over a 12month period (Fonarow et al., 2010).

Chart audit and provider feedback is an effective mechanism for improving provider adherence to standards of care (Ivers et al., 2013) and has specifically been identified as an effective means of improving provider delivery of self-care education (Okelo et al., 2014). The IMPROVE HF registry demonstrated suboptimal delivery of self-care education to individuals with HF (Fonarow et al., 2010) and provides a framework for chart audit guidelines informed by the HFSA and ACCF/AHA (Lindenfeld et al., 2010; Yancy et al., 2013). Ivers et al., (2013) identified that chart audit and provider feedback is most successful when the current standard of practice is suboptimal, provided by a colleague, delivered in written and verbal formats on a continuous monthly basis, and incorporates straightforward goals and plan. 
IMPROVE HF SELF-CARE EDUCATION DELIVERY

The purpose of this review was to inform the identified clinical problem and to identify successful interventions, specific goals, objectives, evaluation measures, and outcome measures. Over all, the strength of the evidence is strong (Appendix A). A strong systematic review (Ivers et al., 2013) has concluded that audit and feedback are effective interventions to create practice change. Performance evaluation, including audit and performance feedback interventions, have been adopted and strongly recommended into current ACCF/AHA 2013 HF Management Guidelines. Heart failure self-care education elements have been identified and supported in the literature to improve health outcomes.

Weakness of the evidence was considered. First, the validity of evidence supporting audit and feedback is contingent upon the accuracy and completion of data obtained during chart auditing. Second, one systematic review used to support this proposal included less than fifty-percent RCTs, which may overstate the benefit of the audit and feedback intervention. However, two of the four studies that evaluated audit and feedback effects on provider performance of self-care education were RCTs (Feder, Griffiths, Highton, Eldridge, Spence, \& Southgate, 1995; Schneider, Wensing, Biessecker, Quizler, Kaufmann-Kolle, \& Szecsenyi, 2008). Third, the two strong professional practice guidelines (Lindenfeld et al., 2010; Yancy et al., 2013) lack significant stakeholder involvement in the informative, evaluative, and disseminative process of guideline development. Self-care education for HF is often a nurse-led intervention; therefore there may be weakness, bias, or gaps in the recommendations. However, the guideline does use a strong methodology in collecting and appraising evidence for practice recommendations. 
IMPROVE HF SELF-CARE EDUCATION DELIVERY

This literature review supports the implementation of a chart audit and provider performance feedback intervention aimed at improving cardiology provider delivery of HF self-care education. Quality measurement of provider delivery of HF self-care education is supported (Fonarow et al., 2010). However, performance measurement of HF self-care education delivery is a low priority to cardiology providers compared to other HF quality measures that are recommended to be linked to performance evaluation and incentives (Fonarow et al., 2010). Inaccurate and incomplete HF self-care delivery documentation is a limitation to obtaining valid provider performance measurement. Chart audit and provider performance feedback interventions have demonstrated statistically significant and clinically meaningful improvements in provider performance measures (Ivers et al., 2013).

\section{Theoretical Framework}

The Iowa Model of Evidence-Based Practice (EBP) to Promote Quality Care (Iowa Model) was used as a framework for this project. The Iowa Model provides a systematic framework for translating evidence into clinical practice (Titler, 2001). The Iowa Model is widely used to guide the implementation of practice change (Gordon, Bartruf, Gordon, Lofgren, \& Widness, 2008; Stebral \& Steelman, 2006; Stenger, Montgomery, \& Briesmeister, 2007). Titler (2001) identifies six key elements of successful execution of EBP: 1) Identifying the Trigger 2) Organizational Priority 3) Teamwork 4) Systematic Review of the Evidence 5) Implementing Evidence into Practice and 6) Evaluating Outcomes. Permission was granted by the University of Iowa, Department of Nursing to use the Iowa Model for EBP for the Improvement of Quality Care for this project (Appendix B). 
IMPROVE HF SELF-CARE EDUCATION DELIVERY

\section{Key Elements of the Iowa Model}

Identifying the trigger. The first key element identified in the Iowa Model for implementing EBP is identification of a pertinent problem or knowledge-focused trigger. Problem focused triggers may include identification of a clinical problem supported by risk management, process improvement, and benchmarking or financial data. Knowledge-focused triggers may include new empirical evidence, practice guidelines or philosophies of care (Titler, 2001). The identified problem-focused trigger in this project is that despite evidence supporting provider delivery of HFSC education, only $61 \%$ of adults with HF receive recommended provider-delivered, self-care education. Furthermore, measurement of HFSC education delivery is often not captured in quality analysis.

Organizational priority. The second step in the Iowa Model is to determine if the problem or knowledge-focus trigger is a priority for the organization (Titler, 2001). Identifying high-resource issues and aligning with the organization's mission, values, and strategic plan facilitates support necessary for completion of an EBP project. If the EBP project is not considered a high-priority topic by senior leadership, the Iowa Model does not support moving forward but re-evaluating the organizational priority, triggers, or outcomes (Titler, 2001).

The site for this capstone project was the Centra Medical Group Stroobants Cardiovascular Center (SCC). The mission of Centra Health is "Excellent care- every time” (Centra Health, 2009). Centra Health has a vision to be "the first choice for our patients, physicians, employees and community” (Centra Health, 2009). Centra Health values patient-centered care, respect and compassion, quality and service, teamwork, 
IMPROVE HF SELF-CARE EDUCATION DELIVERY

continuous learning and improvement, community health involvement, and financial stewardship (Centra Health, 2009). Furthermore, the Centra Medical Group SCC is seeking Advanced Heart Failure Certification from the Joint Commission. Advanced HF Certification is a provided through a partnership between the Joint Commission and the American Heart Association. Strategies promoted to improve HF outcomes are targeted benchmarks for the Advanced HF Certification. Heart-failure self-care education, provider performance, and quality improvement are fundamental elements in the path toward Advanced HF Certification. The aim of this capstone project, improving provider delivery of HF self-care education utilizing a chart audit and provider performance delivery intervention was congruent with the mission, vision, and values of Centra Health and the Centra Medical Group SCC.

The vision for Nursing at Centra is that their nurses "will be known as national leaders for using evidence based practice to provide excellence in patient care and for creating and maintaining an environment for excellence in nursing practice” (Centra Health, 2009). The Iowa Model has been adopted by the Centra Health, Division of Nursing as a framework for implementing evidence into practice. Underpinning this capstone project with the Iowa Model aligned with Centra Nursing trajectory to elevate Centra nurses to the forefront of national professional leaders equipped for excellence in nursing practice.

Form a Team. The third step in the Iowa Model of EBP is to form a team. The team assumes responsibility for evaluating the evidence and developing an implementation and evaluation plan. Titler (2001) emphasizes the importance of enlisting interested key stakeholders as part of the team. Enlisting interested team 


\section{IMPROVE HF SELF-CARE EDUCATION DELIVERY}

members who can capture the essential skills, organizational astuteness and impact required to successfully complete an EBP project is a key element of the Iowa Model. A team was formed to develop and implement the proposed project. The project leader along with a Capstone Project Committee identified a trigger, analyzed organizational priority, evaluated current evidence, and developed a project implementation and evaluation plan. The project leader surveyed SCC for interested and experienced providers and included providers who have a special interest related to HF performance improvement to participate in this project.

Evaluate the evidence. The forth step in the Iowa Model is to evaluate the evidence. Titler (2001) suggest that all members of the team be involved in the evaluating the evidence for the purpose of understanding the scientific underpinning for supporting implementation of the new EBP change. The team determined there was a sufficient knowledge base to support translating the evidence into practice.

Translate evidence into practice. The fifth step in the Iowa Model is to pilot the change in practice. Piloting the change in practice is a multi-step process and involves the team implementing the EBP change. The team is responsible for selecting the change outcomes to be achieved, collecting baseline data, designing and implementing the EBP guidelines, evaluating process and outcomes, and modifying the EBP guideline based upon the process and outcomes evaluation (Titler, 2001). The project leader provided key stakeholders with the project outcomes and elicited input to guide the recommended practice changes and desired outcomes.

Evaluate Outcomes. The sixth step in the Iowa Model for EBP is to evaluate the outcomes for the purpose of deciding if the change is appropriate for adoption into 


\section{IMPROVE HF SELF-CARE EDUCATION DELIVERY}

practice. The investigator conducted data analysis and presented outcomes to key stakeholders, the project team, and to the cardiology group. The team will evaluate the project outcomes to determine if the project was useful for modification and implementation to fit the goals of the practice in improving HFSC education delivery and documentation. The team is now charged with developing a sustainability plan that will include monitoring and analyzing the structure, process, and outcomes data on a continual basis (Titler, 2001).

\section{Design}

\section{Project}

The purpose of this capstone project was to implement and evaluate a chart audit and provider performance feedback intervention directed at improving provider delivery of HFSC education. The aim of this project was to promote a practice change among cardiology providers targeted to improving HFSC education delivery to adults with HFrEF. The project design is constructed upon literature synthesis and underpinned by the Iowa Model.

A team was formed according to the Iowa Model. The project leader presented the project proposal to the capstone committee and elicit input for the purpose of refining the project. The project leader proposed to conduct a baseline (pre-intervention) chart audit and performance analysis aimed at evaluating provider delivery of HFSC education. Individual cardiology providers were given a printed report that detailed the individual provider's performance and the aggregate performance. Pre-intervention aggregate provider performance was presented verbally with a detailed PowerPoint presentation to the cardiology group during a monthly staff meeting. Thirty-days after providing 
IMPROVE HF SELF-CARE EDUCATION DELIVERY

baseline provider performance feedback, post-intervention chart audit and performance analysis evaluating provider delivery of HFSC education was conducted. Individual cardiology providers were given a post-intervention printed feedback report that detailed the individual cardiology provider and aggregate performance report that compared preintervention and post-intervention performance. Post-intervention aggregate provider performance feedback was presented verbally along with a detailed PowerPoint to cardiology providers during a monthly staff meeting.

Cardiology providers were asked to complete a provider satisfaction questionnaire exploring provider satisfaction with quality measurement of HFSC education, provider performance feedback process aimed at improving HFSC education delivery, individual performance feedback and aggregate performance feedback. The project leader then presented outcomes and process evaluations to the project team and the team will determine the implications for future practice.

\section{Setting}

The site for this capstone project was the Centra Medical Group, Stroobants’ Cardiovascular Center located in Lynchburg, Virginia. The proposed project was presented to the Medical Director and verbal and written support for this project was obtained (Appendix H: Letter of Support).

\section{Feasibility Analysis}

The project leader has determined the personnel, material, equipment, and supplemental resources as essential for effective and financially responsible project implementation (Appendix C). The project site has a systems infrastructure supportive of the project and key stakeholder support were sought. The cost-benefit, ethical 
IMPROVE HF SELF-CARE EDUCATION DELIVERY

considerations, project timeline have been taken into account to successfully meet the project objectives.

\section{Personnel}

- Expert Committee

- Quality Improvement/Informatics Technology Support (Quality Improvement Coordinator)

- Nursing and Office Administrative Support (Heart Failure Clinic Staff)

\section{Material}

- Chart audit template (Appendix D)

- Provider satisfaction questionnaire (Appendix E)

- Provider feedback report

- HFSC Documentation Template (Appendix F)

- HFSC Master List Template (Appendix G)

- Office Supplies

\section{Equipment}

- Computer

- Centricity EHR

- Excel

- PowerPoint

- SSPS

- Printer

\section{Supplemental Support}

- Conference Room

\section{Cost-Benefit Analysis}

Personnel resources constitute the largest portion of financial resources (Appendix C: Capstone Budget). Given the large volume of patients with HF treated at clinical site, the high-priority of reducing HF related admissions; along with the organizational priority of quality improvement, the cost-benefit ratio is justifiable.

Furthermore, this intervention was substantially supported by strong evidence and driven 
IMPROVE HF SELF-CARE EDUCATION DELIVERY

by professional guidelines. The clinical site in which this project was implemented

conducts chart audit and provider performance feedback of HF core measures aside from HFSC education delivery. The potential benefit associated with improved HF performance measurement and patient health outcomes are significant. If the project is determined to be a priority for SCC, budgeted cost outlined will require organizational justification.

\section{Ethical Considerations}

The project investigators completed research ethics training to ensure protection of human subjects. The final committee-approved project protocol was submitted to and approved by the lead institution, West Virginia University (WVU), Institutional Review Board (IRB). After WVU IRB approval, the protocol was submitted to Centra Health IRB and approved. The project leader created a master list that contained each eligible participating provider name, assigned provider identification code, chart record number, and chart identification code. The master list was created in an Excel spreadsheet and saved as a password-protected PDF and saved on a password-protected, health information and portability accountability act (HIPAA) compliant computer provided by SCC. Data documentation on the chart audit tool and data analysis documents was conducted using de-identified patient information. The co-investigator maintained the master list as a password protected PDF on a password protected HIPAA compliant computer. These records will be maintained for 3 years after completion of the project. There will be no copies made of the master list and the master list will be purged from the computer after 3 years. There will be no patient or cardiology provider identifying information associated with any presentation or publication of this project. 
IMPROVE HF SELF-CARE EDUCATION DELIVERY

\section{Project Phases}

Preparation. Following the Iowa Model, a problem-focused trigger was identified and determined to be a priority for the organization. A team was formed and literature was assembled and synthesized. It was determined that there was a sufficient research to base the practice change. The project proposal was created, submitted, and approved by the capstone committee. The project was presented to and endorsed by the clinical site medical director (Appendix H). The project was submitted to and approved by the institutional review board of record (Appendix I) and the clinical setting institutional review board (Appendix J). Statistical data files were created.

Implementation. Following the Iowa Model, the audit and feedback intervention was piloted in practice. A 30-day retrospective EHR audit was conducted. Data was collected, entered into the SPSS statistical software, and analyzed. Printed individual provider performance feedback reports and a group feedback verbal and PowerPoint presentations were developed and delivered at a provider group meeting. Thirty days after pre-intervention provider feedback was delivered, a post-intervention 30-day retrospective EHR audit was conducted, and data was collected and analyzed. Postintervention individual provider performance feedback reports and a group feedback verbal and PowerPoint presentation were developed and delivered at a provider group meeting.

Evaluation. Following the Iowa Model, the audit and feedback intervention was evaluated to determine if the change was appropriate for adoption into practice. Postintervention provider satisfaction questionnaires (Appendix E) were distributed at the group provider meeting and anonymously obtained. Provider satisfaction questionnaire 


\section{IMPROVE HF SELF-CARE EDUCATION DELIVERY}

results were analyzed. The project results were discussed with key stakeholders. This capstone project has identified barriers to delivery, documentation, and quality measurement of HFSC education and prompted future practice implications that detailed in the discussion and recommendations section of this manuscript.

\section{Project Objectives}

1. After completion of the EHR audit and provider performance feedback intervention, cardiology providers (aggregate) will improve their performance of HF self-care education delivery to adult patients with HFrEF as evidenced by post-intervention EHR audit demonstrating increased frequency of HF self-care educational element delivery compared to pre-intervention EHR audit.

2. After completion of the EHR audit and provider performance feedback intervention, the individual cardiology providers will improve their performance of HF self-care education deliver to adult patients with HFrEF as evidenced by post-intervention EHR audit demonstrating improved frequency of HF self-care educational element delivery compared to pre-intervention EHR audit.

3. After completion of the EHR audit and provider performance feedback intervention, cardiology providers will report satisfaction with the EHR chart audit and performance feedback process as evidence by self-reported satisfaction questionnaire demonstrating a level of satisfaction of 3 on a 5-point Likert-type satisfaction survey. 
IMPROVE HF SELF-CARE EDUCATION DELIVERY

\section{Evaluation}

A quasi-experimental, one-group pretest/posttest design was used to evaluate the effectiveness of an audit and provider performance feedback intervention on individual and aggregate provider delivery of HFSC education to adults with HFrEF.

The capstone project consisted of two populations. The primary population of interest was cardiology providers. The second population of interest was adult patients with HFrEF. A nonrandom purposive sampling method was used for the primary population of interest, cardiology providers. The clinical setting in which the project was implemented is composed of 36 cardiology providers who conduct office visits in the Lynchburg cardiology clinic $(\mathrm{N}=36)$. Of these cardiology providers, twenty-three $(n=23)$ were eligible for participation. Fifteen of the eligible cardiology providers were medical doctors (MD), 4 of the cardiology providers were nurse practitioners (NP), and 3 of the cardiology providers were physician assistants (PA). Provider inclusion criteria for aggregate performance measures will include:

1) Cardiology Providers (MDs, NPs, or PAs)

2) Provided clinical services in the general cardiology clinic during 30-days chart audit period.

Provider exclusion criteria for aggregate performance measures include:

1) Non-providers (Registered Nurse, Lab, Diagnostic visits).

2) Did not provide clinical services in the general cardiology clinic (Heart Failure Clinic; Electrophysiology Clinic; Pediatric Cardiac Clinic).

3) Heart Failure Clinic Physician, Medical Director.

4) Cardiology NP implementing the capstone project. 
IMPROVE HF SELF-CARE EDUCATION DELIVERY

5) Pediatric cardiology providers.

Patient encounters include cardiology provider-led patient encounters, registered nurse (RN) visits, and diagnostic testing visits. Patient encounters eligible for inclusion in aggregate performance measurement include:

1) Active patients defined as patients who are living, who have HFrEF, and whom a SCC cardiology provider have conducted a patient encounter within the previous 3-years at the SCC-Lynchburg Office.

2) Encountered a cardiology-provider led office visit within the previous 30-days

3) Had a primary or secondary diagnosis of HFrEF (ICD-9 Code 428) or congestive heart failure (ICD-9 Code 425).

4) Age 18-years or older.

Instrumentation and data collection. A 30-day retrospective EHR audit was implemented for pre-intervention and post-intervention evaluation. Data collection proceeded as follows:

1. Chart search method for pre-intervention and post-intervention chart audit per individual provider:

a) Search Centricity EHR for charts completed by provider.

b) Narrow by date range (previous 30-day period).

c) Narrow by patient age in years (18 years or older).

d) Narrowed by document text and/or ICD-9 Code for cardiomyopathy (ICD-9 Code 425) and congestive heart failure (ICD-9 428).

2. The project leader evaluated chart eligibility by confirming a diagnosis of HFrEF (defined as $\mathrm{LVEF}</=35 \%$ ) as a primary or secondary diagnosis. This manual 
IMPROVE HF SELF-CARE EDUCATION DELIVERY

review was necessary because the site EHR system in which data collection occurred did not interface with the current diagnostic electronic record that contains LVEF data.

3. The project leader created 2 master lists. The provider master list (Appendix G: HFSC Master List Template) included the provider name and assigned provider identification code. The chart identification master list included provider identification code, chart record number, and assigned chart identification code. Both master lists were created on a excel document then converted to a password-protected PDF document. The password-protected PDF document has been saved on the project leader's password-word protected, HIPAA compliant computer that is provided by the clinical site.

4. Project leader conducted a chart review and audit of the seven HFSC education elements (Appendix D: Heart Failure Self-Care Electronic Health Record Audit Tool). The data points recorded on the HFSC Electronic Health Record Tool were recorded on a de-identified data on a IBM Statistical Package for Social Sciences (SPSS) dataset (Appendix F: Heart Failure Self-Care Documentation Template Dataset).

6. The project leader will maintain the master list as a password-protected, portable document format (PDF) on a password protected HIPAA compliant computer for 3 years after the completion of this study. There will be no copies made of the master list and the master list will be purged from the computer after 3 years.

Statistical analysis. Statistical analysis was conducted in two phases: pre and post-intervention. Two statistical methods, descriptive and inferential, were used to 
IMPROVE HF SELF-CARE EDUCATION DELIVERY

evaluate objective 1 and objective 2. Descriptive analysis was conducted pre and postintervention to describe the characteristics of the provider (category- MD, NP, or PA), patient sample (age and LVEF), and to determine frequency of individual and aggregate provider delivery of each of the seven HFSC education elements. Nonparametric inferential statistical analysis using McNemar’s Test was used to compare pre- and postintervention independent and aggregate provider delivery of each of the seven HFSC educational elements and HFSC education delivery quality criteria, defined as meeting three or more of the seven educational elements (categorical- met or unmet), were evaluated using univariate descriptive methods.

A provider satisfaction questionnaire was used to explore cardiology provider satisfaction with the EHR audit and provider performance feedback intervention (Appendix E). A literature search was conducted in the Health and Psychosocial Instruments database for a self-report health care provider survey measuring provider satisfaction of an intervention process. However, an appropriate measurement tool was not found. Therefore, the project leader developed a 4-item, Likert-type questionnaire aimed at exploring cardiology provider's attitudes toward chart audit and performance feedback intervention (Appendix E). A literature review was conducted to inform the concepts, constructs, and readability of the survey. The provider satisfaction questionnaire is comprised of 4 items relating to provider satisfaction with the documentation audit as a quality performance measure, feedback delivery type (individual and group) and process. The items are rated on a 5-point scale ranging from 0 (unsatisfied) to 5 (extremely satisfied). The provider satisfaction questionnaire also contained two open-ended questions to allow providers the opportunity to provide 
IMPROVE HF SELF-CARE EDUCATION DELIVERY

additional comments and/or recommendations to improve provider ability to document

HFSC education delivery and how to improve the provider feedback process.

A nonrandom, convenience sampling method was used to survey cardiology

providers who participated in the audit and feedback intervention. Providers who

participated in the audit and feedback intervention were asked to complete the Heart

Failure Self-Care Education Documentation Audit and Feedback Project Provider

Satisfaction Questionnaire (Appendix E). The questionnaire was distributed and

anonymously collected during the post-intervention aggregate provider

performance feedback verbal presentation. Providers who were not present at the group provider meeting were provided with their individual feedback report and provider satisfaction questionnaire. Descriptive analysis (median, mode, range and interquartile range) was conducted to explore provider satisfaction with each of the four items that evaluated provider satisfaction with EHR audit as a quality tool for measuring HFSC education delivery and overall audit and feedback intervention satisfaction with the individual and aggregate performance feedback intervention.

\section{Results}

Pre-Intervention. There were a total of 23 cardiology providers included in this project. Sixteen of the providers were physicians (70\%), four providers were nurse practitioners (17\%), and three providers were physician assistants (13\%). A total of 120 patient encounters were eligible for inclusion in the pre-intervention data analysis. Seventy-four percent of charts were completed by physicians, $22 \%$ were completed by nurse practitioners, and $9 \%$ were completed by physician assistants. 


\section{IMPROVE HF SELF-CARE EDUCATION DELIVERY}

All providers received an individualized feedback report that included individual and aggregate performance results. Fifteen (65\%) were present at the provider meeting in which verbal feedback of aggregate performance was presented. Eight providers (35\%) were not in attendance at the provider meeting in which pre-intervention aggregate performance feedback results were delivered. Of the eight providers, three (38\%) requested and received individual feedback in which pre-intervention individual and aggregate results were discussed.

All patient records included in the pre-intervention analysis had a primary or secondary diagnosis of HFrEF. The LVEF range was $5 \%$ to $35 \%(\mathrm{~m}=23.5 \%)$.

Documented ages of the 120 patients ranged from 38 to $>90$ years $(\mathrm{m}=70$ years; $\mathrm{SD}=$ 12.5). Sixty-two percent of the patients were male and $38 \%$ were female.

Pre-intervention aggregate provider documentation of HFSC educational elements 1 to 7 (Figure 1. Pre-Intervention HFSC Education Documentation) ranged from 3.3\% (HFSC Element 7) to 52.5\% (HFSC Element 4). Heart failure self-care education delivery quality criteria, defined as documentation reflecting delivery of three or more of the seven educational elements was evident in $25 \%$ of pre-intervention charts audited. 
IMPROVE HF SELF-CARE EDUCATION DELIVERY

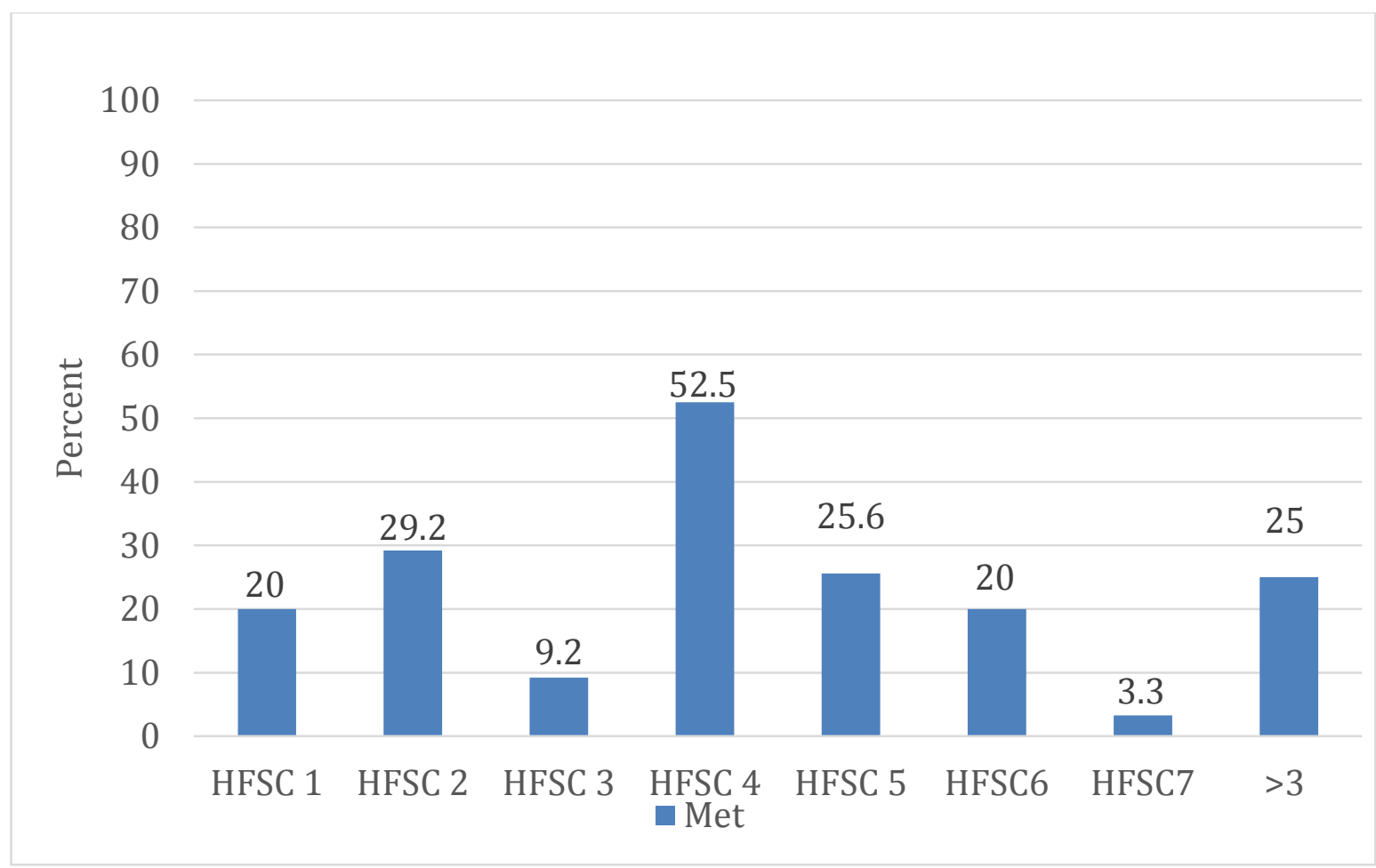

Figure 1. Pre-Intervention HFSC Education Documentation

Post-intervention. A total of 20 providers were included in the postintervention analysis. Three providers, all physicians, were not included in postintervention analysis because they had no eligible charts for analysis. A total of 85 patient encounters were eligible for inclusion in the post-intervention data analysis. Fifty-eight percent of charts were completed by physicians, 22\% were completed by nurse practitioners, and $20 \%$ were completed by physician assistants. All patients had a primary or secondary diagnosis of HFrEF. The LVEF range was 10 to $35 \%$ $(\mathrm{m}=23.5 \%)$. Ages of the 85 patients ranged from 34 to $>90$ years $(\mathrm{m}=70$ years; SD 13.3 years). Fifty-four percent of the patients were female and $46 \%$ were male. Post-intervention aggregate provider documentation of HFSC educational elements 1 to 7 (Figure 2. Post-Intervention HFSC Education Documentation) ranged from $11.8 \%$ (HFSC Element 3) to $64.7 \%$ (HFSC Element 4). Heart failure self-care 
IMPROVE HF SELF-CARE EDUCATION DELIVERY

education delivery quality criteria, defined as documentation reflecting delivery of three or more of the seven educational elements, was evident in $50.6 \%$ of post-intervention charts audited.

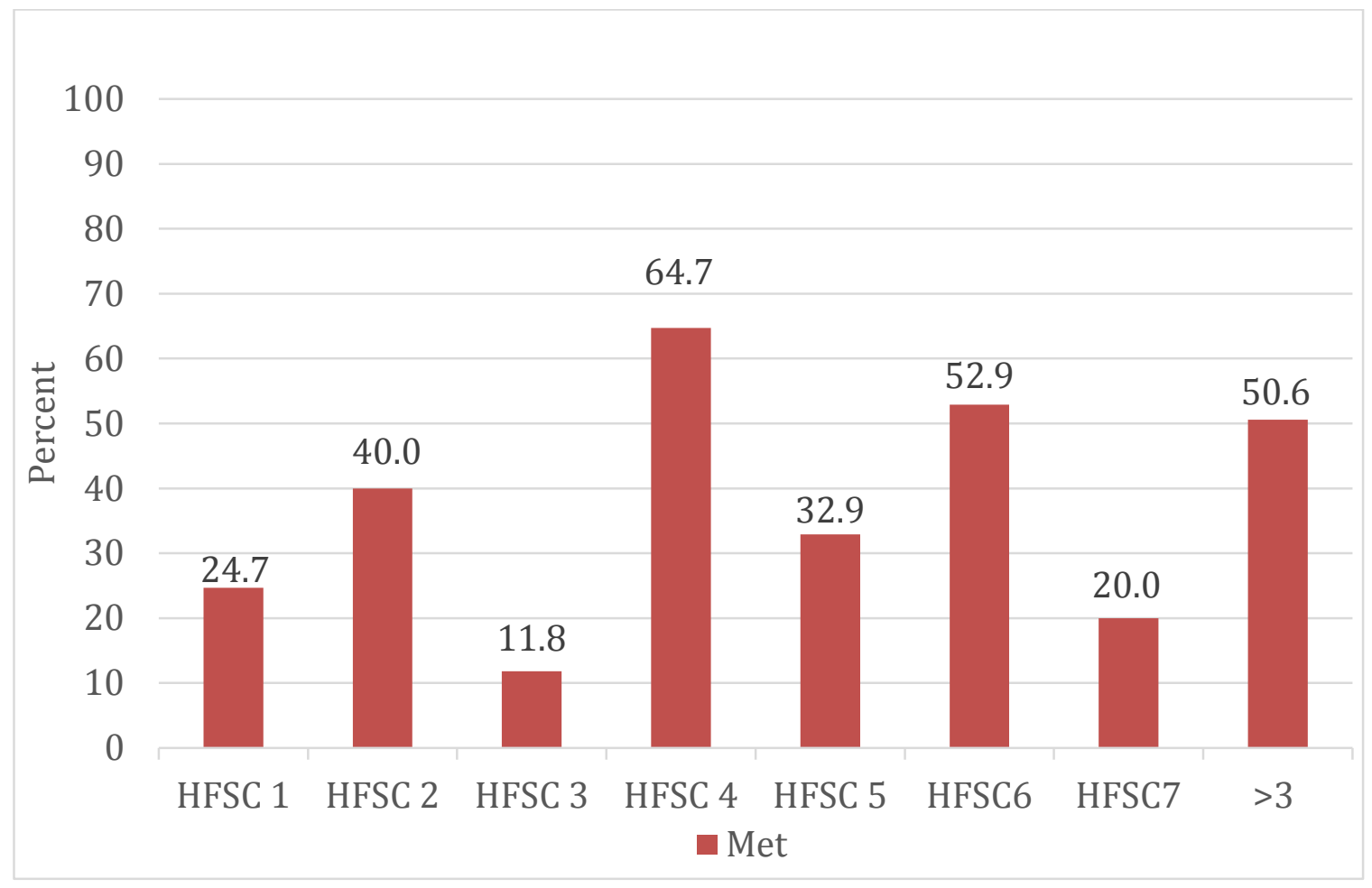

Figure 2. Post-Intervention HFSC Education Documentation

Descriptive statistical analysis was conducted to compare the frequency of preintervention and post-intervention delivery of HFSC educational elements (Figure 3. PreIntervention and Post-Intervention HFSC Education Documentation). McNemar's Test was used to determine if there was statistically significant difference between pre- and post-intervention aggregate provider delivery of each of the seven HFSC educational elements (Table 1. Pre-Intervention and Post-Intervention HFSC Documentation). Post-intervention data analysis demonstrated an improvement in the documentation in all 7 HFSC educational elements and HFSC education delivery quality criteria (Figure 3. Pre-Intervention and Post-Intervention HFSC Education Documentation). 
IMPROVE HF SELF-CARE EDUCATION DELIVERY

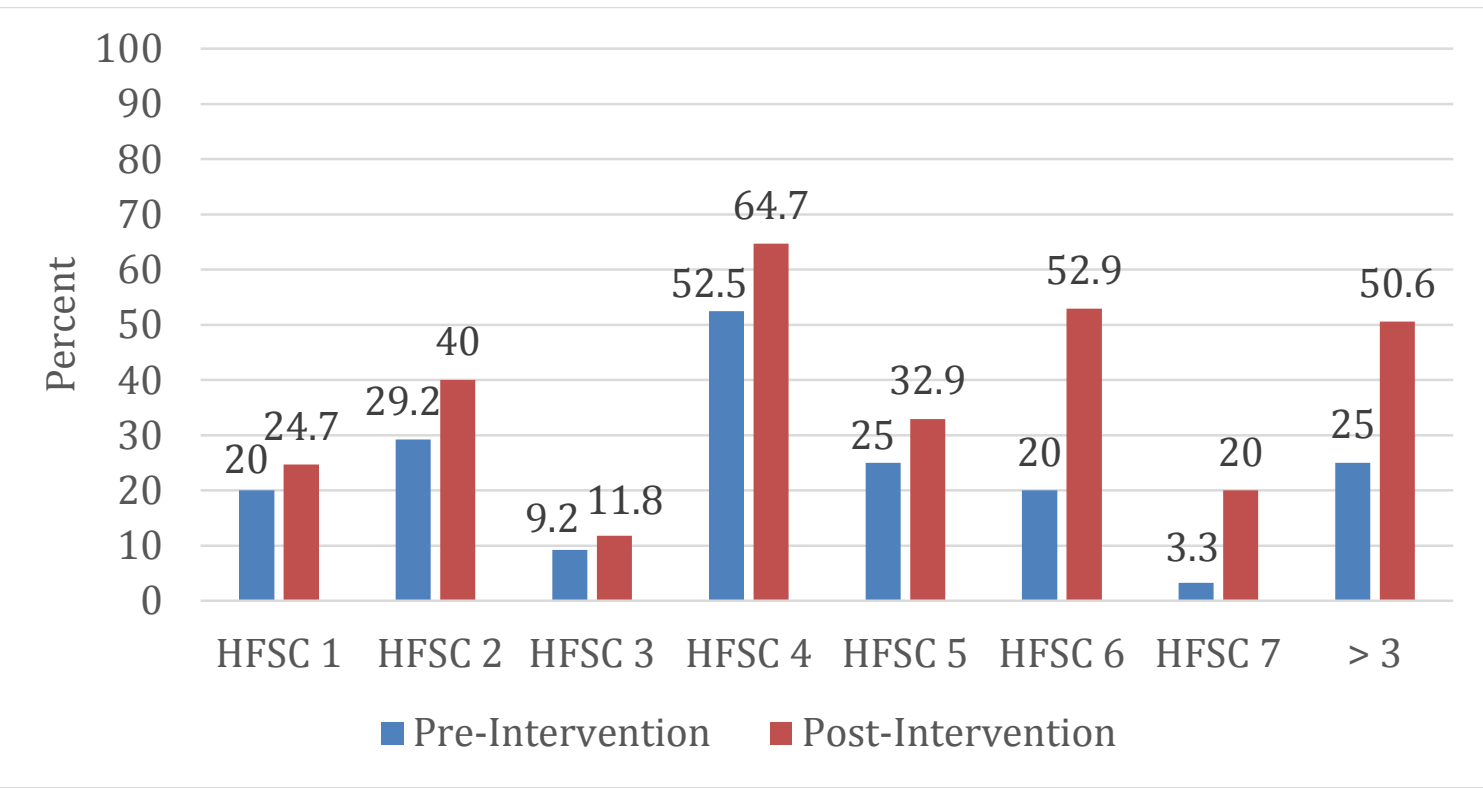

Figure 3. Pre-Intervention and Post-Intervention HFSC Education Documentation

Statistically significant improvements were demonstrated in the documentation of HFSC

education element 2 (29.2\% vs 40\%; p= 0.002), element 5 ( $25 \%$ vs. 32.9\%; p=0.03),

element 6 (20\% vs 52.9\%; p=0.00), element 7 (3.3\% vs 20\%; p=0.04), and HFSC

education quality criteria met (25\% vs 50.6\%; $\mathrm{p}=0.00$ ) (Table 1 . Pre-Intervention and

Post-Intervention HFSC Documentation).

\begin{tabular}{|l|c|c|c|}
\hline \multicolumn{1}{|c|}{ Element } & Pre-Intervention (\%) & Post-Intervention (\%) & $\boldsymbol{p}$ \\
\hline HFSC 1 & 20 & 24.7 & 1.000 \\
\hline HFSC 2 & 29.2 & 40 & .002 \\
\hline HFSC 3 & 9.2 & 11.8 & 1.000 \\
\hline HFSC 4 & 52.5 & 64.7 & .200 \\
\hline HFSC 5 & 25 & 32.9 & .029 \\
\hline HFSC 6 & 20 & 52.9 & .000 \\
\hline HFSC 7 & 3.3 & 20 & .000 \\
\hline $\begin{array}{l}\text { HFSC } \\
\text { Criteria Met } \\
(>3 \text { Elements) }\end{array}$ & 25 & 50.6 & .004 \\
\hline
\end{tabular}

Table 1. Pre-Intervention and Post-Intervention HFSC Documentation 


\section{IMPROVE HF SELF-CARE EDUCATION DELIVERY}

Post-Intervention Individual Results. A total of 19 providers received postintervention feedback. Three providers (MDs) did not have any eligible visits during the 30-day post-intervention time period and one provider (NP) resigned prior to receiving individual feedback. Descriptive statistical analysis was conducted to determine the percentage of individual providers that demonstrated an improvement of HFSC education delivery. Seventy-four percent of providers demonstrated an increase in HFSC education quality criteria met, defined as documentation reflecting delivery of three or more of the seven educational elements.

Provider Satisfaction Questionnaire. Twenty-two providers received the Heart Failure Self-Care Education Documentation Audit and Feedback Project Provider Satisfaction Questionnaire. There was one provider who resigned prior to receiving postintervention feedback. Three providers submitted response the Heart Failure Self-Care Education Documentation Audit and Feedback Project Provider Satisfaction Questionnaire. Due to a small sample size $(n=3)$ the results of this satisfaction questionnaire cannot be considered representative of all providers. All providers who submitted the Heart Failure Self-Care Education Documentation Audit and Feedback Project Provider Satisfaction Questionnaire reported being “extremely satisfied” with all 4 items relating to provider satisfaction with the documentation audit as a quality performance measure, feedback delivery type (individual and group) and process. None of the providers who submitted the satisfaction questionnaire provided additional comments and/or recommendations to improve provider ability to document HFSC education delivery and how to improve the provider feedback process in the two-open ended questions. 
IMPROVE HF SELF-CARE EDUCATION DELIVERY

\section{Discussion and Recommendations}

The Iowa Model was used to guide this project. The Iowa Model provided a framework for identifying inadequate HFSC education delivery at the national level (Fonarow et al., 2010) as a problem-focused trigger. Inadequate delivery of HFSC education was determined to be an organizational priority by a team of expert providers and stakeholders. The Iowa Model emphasizes that all members of the team and key stakeholders evaluate the evidence. There was sufficient evidence to support the delivery, documentation, and quality measurement of HFSC education delivery. The Iowa Model provided a framework for evaluating the project outcomes. The project team, consisting of the project leader and the project committee, evaluated the evidence then the evidence was provided to key stakeholders and to participating cardiology providers. Translating the project into practice is a multi-step process that began as selecting the change outcomes to be achieved, collecting pre- and post-intervention data, designing and delivering individual and aggregate provider feedback, and evaluating processes and outcomes.

The project team and key stakeholders has now been charged with evaluating the outcomes of this project and implications for future practice. Pre- and post-intervention data analysis was considered in the evaluation of provider delivery of HFSC education and the audit and feedback intervention as a method to improve provider delivery of HFSC education. Pre-intervention aggregate provider documentation of HFSC educational elements 1 to 7 (Figure 1. Pre-Intervention HFSC Education Documentation) ranged from 3.3\% (HFSC Element 7) to 52.5\% (HFSC Element 4). Individual and aggregate outcomes suggest that providers prioritize educating 
IMPROVE HF SELF-CARE EDUCATION DELIVERY

patients regarding identification and modification of risk factors associated with $\mathrm{HF}$, such as healthy weight, substance use, and controlling comorbid disease such as hypertension and diabetes mellitus. Pre-intervention HFSC education delivery quality criteria, defined as documentation reflecting delivery of three or more of the seven educational elements was evident in $25 \%$ of pre-intervention charts audited.

Post-intervention aggregate data analysis demonstrated an improvement in the documentation in all 7 HFSC educational elements and HFSC education delivery quality criteria. Statistically significant improvements were demonstrated in the documentation of HFSC education element 2 (29.2\% vs 40\%; $p=0.002$ ), element 5 (25\% vs. 32.9\%; $\mathrm{p}=0.03$ ), element 6 (20\% vs 52.9\%; $\mathrm{p}=0.00)$, element 7 (3.3\% vs $20 \%$; $\mathrm{p}=0.04$ ), and HFSC education quality criteria met (25\% vs 50.6\%; $p=0.00)$. Seventy-four percent of providers demonstrated an increase in HFSC education quality criteria met, defined as documentation reflecting delivery of three or more of the seven educational elements. Post-intervention outcomes suggest that providers prioritized educating patients regarding modification of HF risk factors, specific physical activity or exercise recommendations, and recognition of escalating symptoms of HF volume overload and a plan for responding to symptoms. There is opportunity for improving provider knowledge of and support in the delivery of HFSC education regarding the definition of HF and defining the individual patient's cause of HF, indications and HF medication mechanism of action, and the importance of treatment adherence with strategies to promote adherence.

Potential limitations to provider delivery of HFSC education and the audit and feedback intervention and outcomes have informed the interpretation of the project results and practice initiatives and recommendations. It should be recognized that 
IMPROVE HF SELF-CARE EDUCATION DELIVERY

documentation of HFSC education was used as a proxy for HFSC education delivery. It is implausible to measure actual provider-patient interaction; therefore the HF care delivery system and EHR adequacy for supporting provider delivery and documentation of HFSC education must be considered. It should be recognized that while chart selection was EHR supported and there were objective standards to auditing chart for documentation of HFSC education, the manual chart review that was conducted is subject to auditor interpretation of individual provider delivery of HFSC education elements.

This chart audit and provider feedback project was implemented to evaluate provider delivery of HFSC education, implement a chart audit and feedback aimed at improving HFSC education delivery. The improvement seen in HFSC documentation rates demonstrated during this project are consistent with previous systematic reviews that evaluated the use of chart audit and provider feedback to improve provider performance (Ivers et al., 2012; Okelo et al., 2014). Specifically, Okelo et al (2014) found that four studies aimed at improving provider delivery of self-care education demonstrated significant increases (1-40\%).

While this project was informed by the ACCF/AHA 2012 Heart Failure Guidelines and the IMPROVE HF Registry, the project was designed to evaluate individual and aggregate provider outcomes and potential adoption of the practice change according to the Iowa Model. The IMPROVE HF Registry target population was patients with HFrEF (Fonarow et al., 2007; Fonarow et al., 2010). Therefore, individual patient charts were examined over the course of 1-year to evaluate for documentation of HFSC education delivery. For the purposes of this project utilizing an audit and feedback intervention aimed at improving provider delivery of HFSC 
IMPROVE HF SELF-CARE EDUCATION DELIVERY

education, individual cardiology providers were the target population. Therefore, individual provider charts were audited for evidence of HFSC education delivery over two 30-day periods. The benefit of targeting individual providers was to allow individual providers the opportunity to gain insight into their HFSC education delivery documentation. The subsequent limitation to interpreting aggregate provider HFSC education delivery may be that actual HFSC education delivered to individual patients with HFrEF has underestimated and cannot be quantified.

The outcomes of this project have identified barriers to the delivery, documentation, and quality measurement of HFSC education as an organizational priority and has prompted multi-level practice initiatives and recommendations aimed at decreasing practice barriers and improving HFSC education delivery within the trending value-based provider payment system. While the ACCF/AHA (2013) highlight HFSC education delivery as a quality measure to be evaluated and reported, the evaluation methods are based upon the IMPROVE HF Registry methods that selected outcomes based upon individuals with HFrEF as the target population. The rapid transition to value-based provider payment system is based upon individual provider performance reporting and does not take into consideration the chronology of care an individual with HFrEF receives (Fonarow et al., 2010). Therefore, it is recommended that cardiology practices adopt EHR-savvy methods to demonstrate delivery of HFSC education as a quality measure that align with the transitioning revenue system.

This capstone project provided a foundation for future practice initiatives that are beyond the scope of the project. The first practice initiative aims at improving EHR infrastructure to support provider delivery, documentation, and quality measurement of 
IMPROVE HF SELF-CARE EDUCATION DELIVERY

HFSC education. A team consisting of this project leader, the Quality Measures

Manager, and the Informatics Nurse was formed. The HFSC education essentials were used to develop EHR "point and click" items that allow the provider to populate the patient instruction form with patient-centered HFSC education. This initiative has the potential to benefit individual patients by providing written instructions for their reading and reference. The EHR "point and click" design of this initiative has considered the transition to value-based provider payment system in that each "point and click" is associated with an electronic observation term that is able to be electronically captured for reporting.

Heart failure is a complex, multi-faceted chronic disease and patient self-care education delivery is a complex intervention dependent upon individual patient knowledge and beliefs that requires consideration of the right education, dose, timing, and environment. Potential barriers to provider delivery and documentation of HFSC education include the current provider revenue cycle that emphasizes volume, inadequate EHR infrastructure, and provider knowledge deficit of HFSC education recommendations. Patients encounter challenges to understanding HF and performing HFSC that are based upon health literacy, motivation and self-efficacy, adjustment to health and life-related stresses, social support, and environmental factors (Falvo, 2011). The project site prioritizes HF care delivery in that it has established a HF center of excellence (COE). The HF COE has requested that the project leader assist in the development of a 7-module HF patient education curriculum based upon the 7 HFSC education essentials. Therefore, a team consisting of this project leader, the HF COE 
IMPROVE HF SELF-CARE EDUCATION DELIVERY

Medical Director, and the HF Disease Management Coordinator has been formed to design this initiative.

\section{Attainment of DNP Essentials}

Essential I. The aim of this Doctor of Nursing Practice (DNP) capstone project was to implement and evaluate a chart audit and provider performance feedback intervention to improve provider delivery of HFSC education to individuals with HFrEF. Scientific underpinning for nursing practice is demonstrated by the use of biological, physical, and social science as a foundation for developing and evaluating health care delivery and practice approaches (ANCC, 2006). Essential I has been demonstrated in this project in the use of identification and evaluation of the current literature pertaining to HFrEF epidemiology, pathophysiology, and therapeutic management, identifying an evidence-based practice change to improve provider performance, developing an implementation plan that is based upon an evidence-based practice framework, and evaluating practice performance trends. The implementation and evaluation of this project has provided a foundation for future considerations aimed at improving heart failure outcomes and provider performance measures at the clinical site.

Essential II. According to the ANCC (2006), attainment of Essential II: Organizational and Systems Leadership for Quality Improvement and Systems Thinking is demonstrated by developing, implementing, and evaluating evidence-based health care delivery model aimed at improving population health. The DNP is prepared to utilize organizational and systems models, ethics, nursing, economic, and clinical science knowledge to inform the development and evaluation of health care delivery approaches (ANCC, 2006). Throughout the course of this project, a high priority population (heart 


\section{IMPROVE HF SELF-CARE EDUCATION DELIVERY}

failure population) and specific barriers to quality health outcomes in the heart failure population, specifically the underutilization of HFSC education, have been examined. This project provided the opportunity to demonstrate the ability to lead an organization in the development and evaluation of an evidence-based initiative (provider audit and feedback) aimed at improving health care quality (Ivers et al., 2013).

Furthermore, during the project, Essential II was demonstrated by the analysis of organizational, policy, fiscal, and ethical knowledge. Organization and feasibility analysis provided a framework for developing and implementing the project. Specifically, the project was linked to the organization's mission, vision, and strategic goals utilizing the clinical site’s current resources. Ethical considerations were taken into account when ensuring patient and provider information remains protected during the project. The DNP is prepared to begin analyzing organizational systems and health delivery processes for the main purpose of improving population health outcomes (ANCC, 2006). As a direct result of practicing this Essential II during this project, organizational and community health delivery mechanisms that need to be further analyzed, modified, and evaluated have been identified. For example, after completion of this project, system issues that impede provider ability to deliver HFSC education have been identified. This project also provided the opportunity to explore organizational and systems-based solutions aimed at improving provider delivery and quality measurement of HFSC education.

Essential III. According to the ANCC (2006), attainment of Essential III: Clinical Scholarship and Analytical Methods for Evidence-Based Practice is demonstrated by the ability to engage and lead clinical scholarship at the highest level of 
IMPROVE HF SELF-CARE EDUCATION DELIVERY

nursing practice. Generally, this means analyzing and applying best knowledge to practice and then evaluating the outcomes. Completion of this project provided the opportunity to critically appraise evidence, which laid the foundation for this project. Specifically, this project was aimed at improving provider delivery of HFSC education. Clinical scholarship was the impetus for this project. Current evidence suggest that within cardiology practices at the national level, only $60 \%$ of patients with HF receive adequate HFSC education (Fonarow et al., 2010). After identifying this gap in provider performance at the national level, the need to analyze the clinical site's current practices and outcomes then compare these performance outcomes to best evidence (HFSA guidelines for HFSC education), the national benchmark (Fonarow et al., 2010), become evident. During this project, baseline data was collected in order to identify the gaps in provider delivery of HFSC education key essentials. This data was used to generate provider performance feedback, allowing the cardiology group to examine patterns and outcomes and identify areas for practice improvement (Ivers et al., 2012). Lastly, according to Essential III, the DNP is prepared to disseminate findings of their scholarship to improve healthcare outcomes. Dissemination of project results and recommendations will be occur internally within the clinical site and within the nursing profession at a national conference.

Essential IV. According to the ANCC (2006) the DNP is characterized by their ability to lead the application of information systems to support and improve health care outcomes. Specifically, the DNP is prepared to evaluate and utilize the utilize information systems to evaluate and improve health care outcomes (ANCC, 2006). Generally, the DNP will be able to design, implement, and/or evaluate technology 
IMPROVE HF SELF-CARE EDUCATION DELIVERY

systems across the health care system to include the examination of technical, legal, ethical, and/or organizational information. Throughout the course of the project, Essential IV has been demonstrated. During this project, technical and ethical issues surrounding the use of technology in the care system has been considered. During the course of developing this project, an EHR system's capacities have been evaluated and an evidence-based project has been designed that integrated data mining processes in order to assess provider delivery of HFSC education delivery. Furthermore, the clinical site EHR performance capabilities were considered as a means to improve provider's ability to more effectively and efficiently deliver and document HFSC education.

Essential V. According to the ANCC (2006) the DNP is characterized by the ability to lead in the design, influence, and implementation of health policy within the nursing profession and health care system (Essential V). The DNP is prepared to analyze, develop, and demonstrate leadership in the implementation of institutional health policy (ANCC, 2006). Underpinned by scientific and ethical principles, this projected provided the opportunity to demonstrate the ability to influence and recommend institutional policies aimed at improving provider delivery of HFSC education as a quality measure. Furthermore, this project has increased the knowledge of heart failure care delivery and the use of audit and feedback intervention, which allows for the education of policy makers at the institutional and regional levels.

Essential VI. The DNP is uniquely positioned to successfully lead interprofessional teams for improving patient and population health (ANCC, 2006). Essential VI was demonstrated during the course of this project. First, the Iowa Model of Evidence Based Practice was used as a conceptual framework. The Iowa Model provides 
IMPROVE HF SELF-CARE EDUCATION DELIVERY

a framework which identifies interprofessional collaborates as key stakeholders to inform, implement, and evaluate an evidence-based practice change. Interprofessional leadership and collaboration with physicians, administrators, information technologist, registered nurses, nurse practitioners, and physician assistants was required to analyze the organizational resources and current practices and design, implement, and evaluate the project. Furthermore, developing and delivering provider performance feedback required effective leadership and communication skills.

Essential VII. ANCC (2006) has set forth the standard that the DNP will be prepared to "engage in leadership to integrate and institutionalize evidence-based clinical prevention and population health services for individuals, aggregates, and populations”. During the course this project, epidemiological and biostatistical data of aggregatepatients with heart failure has been analyzed. While it was discovered that heart failure is not the most prevalent disease plaguing society, it is by far the most costly due to patients, communities, and organizations (Go et al., 2013). The project experience provided the opportunity to identify inadequate health care delivery to an aggregate. Only $60 \%$ of individuals with heart failure receive minimal self-care education (Fonarow, 2010). Self-care education is a complex intervention and care models to improve delivery were examined. This project included two populations: the heart failure patient aggregate and the cardiovascular provider. While considering the self-management education needs of the heart failure aggregate, community, environmental, and cultural dimensions of health were analyzed. This project aim was to improve provider delivery of HFSC education. While considering an intervention aimed at improving provider 
IMPROVE HF SELF-CARE EDUCATION DELIVERY

delivery of self-care education, care delivery models and strategies aimed at improving provider delivery of heart failure self- care education were evaluated.

Essential VIII. This project has provided the opportunity to identify a nursesensitive clinical practice issue and design, implement and evaluate an evidence-based intervention aimed at improving health outcomes. HFSC education that has been suggested to reduce hospitalization admissions and improved quality of life. Heart failure self-care education has been demonstrated to improve health care outcomes, however the IMPROVE HF Registry demonstrated that only $60 \%$ of individuals of HF receive minimal HFSC education. This project was designed to use a provider audit and feedback intervention aimed at improving provider behavior. During this project, aspects of HFSC education delivery and impacting factors such as population demographics on the clinician practice and the organization including economic, and organizational policy were analyzed and evidence-based recommendations to improve outcomes were designed, implemented, and disseminated, in order to improve HF outcomes. 
IMPROVE HF SELF-CARE EDUCATION DELIVERY

\section{References}

Appalachian Regional Commission. (2008). Map of counties in the Appalachian Region. Obtained from www.arc/gov/appalachian_region/MapofAppalachia.asp.

Baker, R., Fraser, R.C., Stone, M., Lambert, P., Stevenson, K., \& Shiels, C. (2003). Randomized controlled trial of the impact of guidelines, prioritized review criteria and feedback on implementation of recommendations for angina and asthma. British Journal of General Practice, 53 (489), 284-291.

Boren, S.A., Wakefield, B.J., Gunlock, T.L., \& Wakefield, D.S. (2009). Heart failure self-management education: A systematic review of the evidence. International Journal of Evidence Based Healthcare, 7, 159-68.

Calvillo-King, L., Arnold, D., Eubank, K.J., Lo, M., Yunyongying, P., Stieglitz, H.,... Halm, E.A. (2013). Impact of social factors on risk or readmission or mortality in pneumonia and heart failure: A systematic review. Journal of General Internal Medicine, 28 (2), 269-282.

Catterjee, K. (2005). Neurohormonal activation in congestive heart failure and the role of vasopressin. American Journal of Cardiology, 95, 8B-13B.

Center for Disease Control (2010). Heart Failure Facts. Obtained from http://www.cdc.gov.

Centra Health. (2009). About Centra: Mission and values. Retrieved from http://www.centrahealth.com/about-centra/mission-and-values.

Coleman, C.L., Reddy, P., Laster-Bradley, N.M., Dorval, S., Munagala, B., \& White, C.M. (2003). Effect of practitioner education on adherence to asthma treatment guidelines. Annals of Pharmacotherapy, 37 (7-8), 956-61. 
IMPROVE HF SELF-CARE EDUCATION DELIVERY

Colucci, W.S., \& Braunwald, E. (2005). Pathophysiology of heart failure. In D.P Zipes, P. Libby, R.O. Bonow \& E. Braunwald (Eds.), Braunwald's heart disease: A textbook of cardiovascular medicine ( $7^{\text {th }}$ Ed.), (pp. 509-538). Philadelphia, PA: Elsevier.

Feder, G., Griffiths, C., Highton, C., Eldridge, S., Spence, M., \& Southgate, L. (1995). Do clinical guidelines introduced with practice-based education improve care of asthmatic and diabetic patients? A randomized controlled trial in general practices in east London. British Medical Journal, 311 (7081), 1473-78.

Falvo, D.R. (2011). Effective patient education: A guide to increased adherence (4 $4^{\text {th }}$ ed.). Sudbury, MA: Jones and Bartlett Publishers.

Fonarow, G.C., Albert, N.M, Curtis, A.B., Stough, W.G., Gheorghiade, M., Heywood, J.T., ...Yancy, C.W. (2007). Improving the use of evidence-based heart failure therapies in the outpatient setting: the IMPROVE HF performance improvement registry. American Heart Journal, 154, 12-38.

Fonarow, G.C., Albert, N.M, Curtis, A.B., Stough, W.G., Gheorghiade, M., Heywood, J.T., ...Yancy, C.W. (2010). Improving evidence-based care for heart failure in outpatient cardiology practices: Primary results of the registry to improve the use of evidence-based heart failure therapies in the outpatient setting (IMPROVE HF). Circulation, 122: 585-96.

Giannessi, D., Del Ry, S., \& Vitale, R.L. (2001). The role of endothelins and their receptors in heart failure. Pharmacology Research, 43, 111-26. 
IMPROVE HF SELF-CARE EDUCATION DELIVERY

Go, A.S., Mozaffarian, D., Roger, V.L., Benjamin, E.J., Berry, J.D., Borden, W.B., ...Turner, M.B. (2013). Heart disease and stroke statistics- 2013 update: a report from the American Heart Association. Circulation, 127, e6-e245.

Gordan, M., Bartruff, L., Gordon, S., Lofgren, M., \& Widness, J. (2008). How fast is too fast? A practice change in umbilical arterial catheter blood sampling using the Iowa model for evidence-based practice. Advances in Neonatal Care, 8 (4), 198-207.

Heidenreich, P.A., Trogdon, J.G., Khavjou, O.A., Butler, J., Dracup, K., ...Woo, Y.J. (2011). Forecasting the future of cardiovascular disease in the United States: A policy statement fro the American Heart Association. Circulation, 123, 933-44.

Hersh, A.,M., Masoudi, F.A., \& Allen, L.A. (2013). Postdischarge environment following heart failure hospitalization: Expanding the view of hospital readmission. Journal of the American Heart Association. Doi:

10.1161/JAHA.113.000116.

Huttlinger, K.W., \& Purnell, L.D. (2008). People of Appalachian heritage. In L.D.

Purnell \& B.J. Paulanka (Eds), Transcultural health care: A culturally competent approach ( $3^{\text {rd }}$ ed.). Philadelphia, PA: F.A. Davis.

Huttlinger, K., Schaller-Ayers, J., \& Lawson, T. (2004). Health care in Appalachia: A population-based approach. Public Health Nursing, 21 (2), 103-110.

Ivers N, Jamtvedt G, Flottorp S, Young JM, Odgaard-Jensen J, ..., Oxman AD. (2012). Audit and feedback: effects on professional practice and healthcare outcomes. Cochrane Database of Systematic Reviews. DOI:

10.1002/14651858.CD000259.pub3. 
IMPROVE HF SELF-CARE EDUCATION DELIVERY

Jamtvedt, G., Young, J.M., Kristoffersen, D.T., OBrian, M.A., \& Oxman, A. (2006).

Audit and feedback: Effects on professional practice and health care outcomes. Cochrane Database of Systematic Reviews, DOI:

10.1002/14651858.CD000259.pub2.

Katz, A. (2011). Heart failure. In A. Katz (Ed.), Pathophysiology of the heart (pp. 510547). Philadelphia, PA: Lippincott Williams \& Wilkins.

Larrabee, J.H. (2009). Nurse to nurse: Evidence-based practice. New York, NY: McGraw-Hill.

Lee, W.C., Chavez, Y.E., Baker, T., \& Luce, B.R. (2004). Economic burden of heart failure: A summary of recent literature. Heart Lung, 33 (6), 362-71.

Lee, C.S., Moser, D.K., Lennie, T.A., \& Riegel., B. (2011). Event-free survival in adults with heart failure who engage in self-care management. Heart Lung, 40 (1), 1220.

Levy, D., Kenchaiah, S., Larson, M.G., Benjamin, E.J., Kupka, M.J., ... Vasan, R.S. (2002). Long-term trends in the incidence of and survival with heart failure. New England Journal of Medicine, 347, 1397-1402.

Liao, L., Anstrom, K.J., Gottdiener, J.S., Pappas, P.A., Whellan, D.J., ...Jollis, J.G. (2007). Long-term costs and resource use in elderly participants with congestive heart failure in the Cardiovascular Health Study. American Heart Journal, 153 (2), 245-52.

Liao, L., Jollis,, J.G., Anstrom, K.J., Whellan, D.J., Kitzman, D.W.,... Gottdiener, J.S. (2006). Costs for heart failure with normal vs reduced ejection fraction. Archives of Internal Medicine, 166 (1), 112-8. 
IMPROVE HF SELF-CARE EDUCATION DELIVERY

Lindenfeld, J., Albert, N.M., Boehmer, J.P., Collins, S.P., Ezekowitz, J.A., ... Walsh, M.N. (2010). Nonpharmacologic management and health care maintenance in patients with chronic heart failure: HFSA 2010 comprehensive heart failure practice guidelines. Journal of Cardiac Failure, 16 (6), e61-72.

Linne, A.B., Liedholm, H., Jendteg, S., Israelsson, B. (2000). Health care costs of heart failure: Results from a randomized study of patient education. European Journal of Heart Failure, 2 (3), 291-7.

Marciniak, T.A., Ellerbeck, E.F., Radford, M.J, et al. (1998). Improving the quality of care for Medicare patients with acute myocardial infarction: results from the Cooperative Cardiovascular Project. Journal of the American Medical Association, 279, 1351-7.

McDonald, K. (2011). Disease management programs for heart failure. Current Treatment Options in Cardiovascular Medicine, 12 (6), 578-86.

McGarvey, E.L., Leon-Verdin, M., Killows, L.F., Guterbock, T., \& Cohn, W.F. (2011). Health disparities between Appalachian and non-Appalachian counties in Virginia USA. Journal of Community Health, 36 (3), 348-356.

National Heart, Lung, and Blood Institute. (2006). Incidence and prevalence: 2006 Chart book on cardiovascular and lung disease. Bethesda, MD: National Heart, Lung, and Blood Institute.

Nishikimi, T., Maeda, N., \& Matsuoka, H. (2006). The role of natriuretic peptides in cardioprotection. Cardiovascular Research, 69, 318-28.

Okelo, S.O., Butz, A.M., Sharma, R., Diette, G.B., Pitts, S.I., ...Robinson, K.A. (2013). Interventions to modify health care provider adherence to asthma 
IMPROVE HF SELF-CARE EDUCATION DELIVERY

guidelines: A systematic review. Pediatrics, 132 (5), 517-534.

Physician Consortium for Performance Improvement (2012). Heart failure performance measurement set.

Richman, M.J., \& Poltawsky, J.S. (2000). Partnership for excellence in asthma care: Evidence-based disease management. Study of Health Technology Information, 76, 107-121.

Roger, V.L., Weston, S.A., Redfield, M.M., Hellermann-Homan, J.P., Killian, J., ...Jacobsen, S.J. (2004). Trends in heart failure incidence and survival in a community-based population. Journal of the American Medical Association, 292, 344-350.

Saraiva, R.M., \& Hare, J.M. (2006). Nitric oxide signaling in the cardiovascular system: Implications for heart failure. Current Opinions in Cardiology, 21, 221-8.

Schneider, A., Wensing, M., Biessecker, K., Quinzler, R., Kaufmman-Kolle, P., \& Szecsenyi, J. (2008). Impact of quality circles for improvement of asthma care: Results of a randomized controlled trial. Journal of Evaluation of Clinical Practice, 14 (2), 1850190.

Scottish Intercollegiate Guidelines Network (2008). Retrieved from http://www.sign.ac.uk/guidelines/audit/index.html.

Stebral, L., \& Steelman, v. (2006). Double-gloving for surgical procedures: An evidence-based practice project. Perioperative Nursing Clinics, 1 (3), 251260.

Stenger, K., Montgomer, L., \& Briesemeister, E. (2007). Creating a culture of change 
IMPROVE HF SELF-CARE EDUCATION DELIVERY

through implementation of a safe patient handling program. Critical Care Nursing Clinics of North America, 19 (2), 213-222.

Stroobants’ Cardiology. (2014). Centra cardiology services: Stroobants’ Cardiovascular. Retrieved from http://www.stroobantscardiovascular.com

Rajagopalan, S., \& Pitt, B. (2003). Aldosterone as a target in congestive heart failure. Medical Clinic of North America, 87, 441-57.

Riegal., B., Carlson, B., Moser, D.K., Sebern, M., Hicks, F.D., \& Roland, V. (2004). Psychometric testing of the self-care of heart failure index. Journal of Cardiac Failure, 10, 350-360.

Roger, V.L., Go, A.S., Lloyd-Jones, D.M., Benjamin, E.J., Berry, J.D...Turner, M.B. (2012). Heart disease and stroke statistics- 2012 update. Circulation, 125 (1), e2220.

The Robert Wood Johnson Foundation (2012). County Health Rankings: Virginia. Retrieved from http://www.countyhealthrankings.org.

Titler, M.G., Kleiber, C., Steelman, V.J., Rakel, B.A., Budreau, G., Everett, L.Q., ...Goode, C.J. (2001). The Iowa Model of Evidence-Based Practice to Promote Quality Care. Critical Care Nursing Clinics of North America, 13 (4), 497-509.

United States Census Bureau (2012). State and county quick facts: Virginia. Retrieved from http://quickfacts.census.gov/qfd/states/51000.html on September 1, 2013.

Whellan, D.J., Hasselblad, V., Peterson, E., O’Connor, C.M., Schulman, K.A. (2005). Metaanalysis and review of heart failure disease management randomized controlled clinical trials. American Heart Journal, 149 (4), 722-9. 
IMPROVE HF SELF-CARE EDUCATION DELIVERY

Wewers, M.E., Katz, M., Fickle, D., \& Paskett, E.D. (2006). Risky behaviors among Ohio Appalachian adults, Prevention of Chronic Disease. Retrieved from http://www.cdc.gov/pcd/issues/2006/oct/06_0032.htm

Yancy, C.W., Jessup, M., Bozkurt, B., Masoudi, F.A., Butler, J...Wilkoff, B.L. (2013). 2013 ACCF/AHA guideline for the management of heart failure. Journal of the American College of Cardiology, doi: 10.1016/j.jacc.2013.05.019. 
IMPROVE HF SELF-CARE EDUCATION DELIVERY

\section{Appendix A}

\begin{tabular}{|l|c|l|l|}
\hline \multicolumn{1}{|c|}{ Reference } & \multicolumn{1}{|c|}{$\begin{array}{c}\text { Level of } \\
\text { Evidence }\end{array}$} & \multicolumn{1}{|c|}{ Type of Evidence } & \multicolumn{1}{c|}{ Comment } \\
\hline Ivers et al. (2012) & 1a & SR of RCTs & $\begin{array}{l}\text { Effects of audit and feedback on } \\
\text { outcomes }\end{array}$ \\
\hline Yancy et al. (2013) & 1a & $\begin{array}{l}\text { Systematic Clinical Practice } \\
\text { Guideline }\end{array}$ & $\begin{array}{l}\text { ACC/AHA HF Practice } \\
\text { Guidelines 2013 Update }\end{array}$ \\
\hline $\begin{array}{l}\text { Lindenfeld et al } \\
\text { (2010) }\end{array}$ & 1a & $\begin{array}{l}\text { Systematic clinical practice } \\
\text { guideline }\end{array}$ & $\begin{array}{l}\text { HFSA Practice } \\
\text { Recommendations for the } \\
\text { nonpharmacological treatment of } \\
\text { HF }\end{array}$ \\
\hline Fonarow et al. & 1c & $\begin{array}{l}\text { Prospective, quality control trial } \\
\text { without randomization. }\end{array}$ & $\begin{array}{l}\text { Large multi-site controlled trial, } \\
\text { measured HF self-care education } \\
\text { delivery. Limitations- included } \\
\text { multiple interventions. }\end{array}$ \\
\hline Oke10) & 2a et al. (2014) & SR & 35 RCTs \\
\hline
\end{tabular}

* Larrabee, J.H. (2009). Nurse to nurse: Evidence-based practice. New York: McGraw-Hill. 
IMPROVE HF SELF-CARE EDUCATION DELIVERY

\section{Appendix B}

West Virginia University Mail - Permission to Use and/or Reproduce The Iowa Model 10/18/14, 9:23 PM

\section{Permission to Use and/or Reproduce The lowa Model}

\section{Kimberly Jordan - University of Iowa Hospitals and Clinics}

<noreply@qemailserver.com> Wed, Jul 9, 2014 at 8:28 PM Reply-To: Kimberly Jordan University of Iowa Hospitals and Clinics < kimberly-jordan@uiowa.edu> To:

dmurph10@mix.wvu.edu

You have permission, as requested today, to review/use The Iowa Model of Evidence-

Based Practice to Promote Quality Care (Titler et al., 2001). Click the PDF file below to download the model.

Copyright of the Iowa Model of Evidence-Based Practice to Promote Quality Care will be retained by The University of Iowa Hospitals and Clinics.

Permission is not granted for placing the Iowa Model on the internet (world-wide web). Please click on this link The Iowa Model to obtain a copy of the model.

In written material, please add the following statement:

Used/Reprinted with permission from the University of Iowa Hospitals and Clinics and Marita G. Titler, PhD, RN, FAAN. Copyright 1998. For permission to use or reproduce the model, please contact the University of Iowa Hospitals and Clinics at (319)384-9098. If you have questions, please contact Kimberly Jordan at 319-384-9098_or kimberlyjordan@uiowa.edu. 
IMPROVE HF SELF-CARE EDUCATION DELIVERY

Appendix C

\section{Budget Form}

\begin{tabular}{|c|c|c|c|c|c|c|c|}
\hline \multicolumn{7}{|c|}{ Project Budget } & $\begin{array}{r}\text { In-kind } \\
\text { donations }\end{array}$ \\
\hline Name & Title & $\begin{array}{l}\text { Total } \\
\text { Salary }\end{array}$ & $\begin{array}{c}\text { Total } \\
\%\end{array}$ & $\begin{array}{l}\text { Total } \\
\text { Salary }\end{array}$ & Fringe & $\begin{array}{c}\text { Total } \\
\text { salary + } \\
\text { fringe } \\
\end{array}$ & \\
\hline $\begin{array}{l}\text { Cardiology } \\
\text { NP }(0.5 \\
\text { day x } 4 \\
\text { weeks) }\end{array}$ & NP & $100,000.00$ & 0.02 & $\$ 1,000$ & $\$ 200$ & $\$ 2,400$ & $\begin{array}{r}\$ 1,200 \\
\text { (DNP } \\
\text { Student) }\end{array}$ \\
\hline \multicolumn{4}{|c|}{ Subtotal for Personnel: } & $\$ 1,000$ & $\$ 200$ & $\$ 2,400$ & $\$ 1,200$ \\
\hline \multicolumn{7}{|c|}{$\begin{array}{l}\text { Justify: NP Investigator to provide service as part of capstone project } \\
\text { requirements. } \\
\text { Additional proposed project cost will be justified by SCC upon } \\
\text { determining organizational priority. }\end{array}$} & $\begin{array}{l}-1,200 \\
\text { DNP } \\
\text { Student } \\
\text { Time }\end{array}$ \\
\hline \multicolumn{8}{|c|}{ Supplies (educational materials, office supplies, cost of copying) } \\
\hline \multicolumn{5}{|c|}{ SPSS } & $\$$ & $\$ 100$ & $\begin{array}{r}100 \text { (DNP } \\
\text { Student) }\end{array}$ \\
\hline \multicolumn{5}{|c|}{ Office Supplies } & $\$$ & $\$ 50$ & $\begin{array}{r}50 \\
\text { (DNP } \\
\text { Student) }\end{array}$ \\
\hline \multicolumn{6}{|c|}{ Supplies Subtotal: } & $\$ 150$ & \\
\hline \multicolumn{7}{|c|}{$\begin{array}{l}\text { Justify } \\
\text { Project leader required to purchase SPSS for capstone project and will } \\
\text { justify project office supplies expenses. }\end{array}$} & $\begin{array}{l}-100 \text { LU } \\
-50 \\
\text { (Student } \\
\text { Expense) }\end{array}$ \\
\hline \multicolumn{8}{|c|}{ Marketing (advertising, fliers, brochures) NA } \\
\hline & & & & & $\$$ & $\$$ & \\
\hline \multicolumn{6}{|c|}{ Other Subtotal: } & $\$$ & \\
\hline \multicolumn{6}{|c|}{ Total Direct Costs } & $\$ 1,350.00$ & \\
\hline \multicolumn{6}{|c|}{ Total Expenses: } & $\$ 1,350.00$ & $-\$ 1,350$ \\
\hline
\end{tabular}


IMPROVE HF SELF-CARE EDUCATION DELIVERY

Appendix D

\begin{tabular}{|l|l|l|l|}
\hline $\begin{array}{l}\text { Chart ID } \\
\text { Code }\end{array}$ & LVEF (\%) & \\
\hline $\begin{array}{l}\text { Patient } \\
\text { Age } \\
\text { (years) }\end{array}$ & & $\begin{array}{l}\text { Provider ID } \\
\text { Code }\end{array}$ & \\
\hline ICD-9 & & $\begin{array}{l}\text { Provider Category } \\
\text { Code }\end{array}$ & \\
\hline
\end{tabular}

Check if heart failure self-care education element documented (written or verbal) to the patient in the patient encounter.

\begin{tabular}{|c|c|c|}
\hline Met & Unmet & Educational Element \\
\hline & & Description of heart failure (Element 1 ). \\
\hline & & $\begin{array}{l}\text { Recognition of escalating symptoms and concrete plan for response to } \\
\text { particular symptoms (Element 2). }\end{array}$ \\
\hline & & $\begin{array}{l}\text { Indication and use of each heart failure pharmacological therapies (Element } \\
\text { 3). }\end{array}$ \\
\hline & & Modification of risk for heart failure progression (Element 4). \\
\hline & & Specific diet recommendation (Element 5). \\
\hline & & Specific activity/exercise recommendation (Element 6). \\
\hline & & $\begin{array}{l}\text { Individualized education addressing the importance of adherence and } \\
\text { behavioral strategies to promote adherence (Element 7). }\end{array}$ \\
\hline
\end{tabular}


IMPROVE HF SELF-CARE EDUCATION DELIVERY

Explanation(s):

1. Description of heart failure (definition of heart failure, linking disease, symptoms, treatment, cause of heart failure.

2. Recognition of escalating symptoms and concrete plan for response to particular symptoms. (Perform daily weights and how to respond to evidence of volume overload; Identify specific symptoms of fatigue, shortness of breath, nocturnal dyspnea, orthopnea, or edema and how to respond.).

3. Indication and use of each heart failure pharmacological therapies (Dosing schedule, basic purpose of specific medication, what to do if dose missed).

4. Modification of risk for heart failure progression (Smoking cessation, Maintain BP in target range, Maintain normal HgA1c (if diabetic), Maintain specific body weight).

5. Specific diet recommendation (Individualized low-sodium diet, Recommendation for alcohol intake).

6. Specific activity/exercise recommendation.

7. Importance of treatment adherence and behavioral strategies to promote. 
IMPROVE HF SELF-CARE EDUCATION DELIVERY

\section{Appendix E}

\section{Heart Failure Self-Care Education Documentation Audit and Feedback Project Provider Satisfaction Questionnaire}

Please respond to the following questions using a scale of 0 to 5 with 0 being not satisfied and 5 being extremely satisfied.

1. How would rate your satisfaction with the Heart Failure Self-Care Education documentation audit evaluating heart failure self-care documentation as a quality performance measurement?
0
1
2
3
4
5

2. How would you rate your satisfaction with the provider feedback delivery process utilized in the Heart Failure Self-Care Documentation Improvement project?
0
1
2
3
4
5

3. How would you rate your satisfaction with receiving individual provider performance feedback regarding delivery of heart failure self-care education?

0

12

3

4

5

4. How would you rate your satisfaction with receiving group performance feedback regarding delivery of heart failure self-care education?

0

1

2

3

4 
5. Please provide any additional comments or recommendations that may improve Heart Failure Self-Care education documentation.

6. Please provide any additional comments or recommendations that may improve the provider performance feedback process. 
IMPROVE HF SELF-CARE EDUCATION DELIVERY

\section{Appendix F}

Heart Failure Self-Care Documentation Spreadsheet Template

\begin{tabular}{|c|c|c|c|c|c|c|c|c|c|c|c|c|c|c|}
\hline \multirow{2}{*}{ 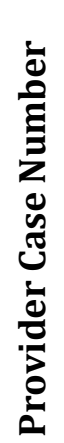 } & \multirow[b]{2}{*}{$\begin{array}{l}0 \\
0 \\
0 \\
0 \\
0 \\
0\end{array}$} & \multirow{2}{*}{ 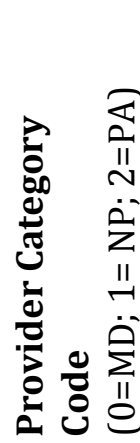 } & \multirow[b]{2}{*}{ 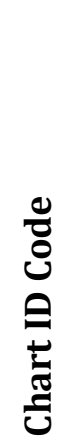 } & \multirow{2}{*}{ 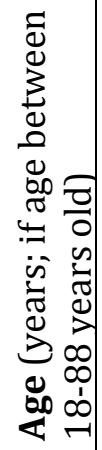 } & \multirow{2}{*}{ 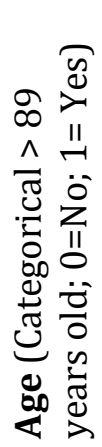 } & & \multicolumn{8}{|c|}{$\begin{array}{l}\text { Heart Failure Self-Care Education Element } \\
\qquad 0=\text { Unmet } 1=\text { Met }\end{array}$} \\
\hline & & & & & & $\underbrace{d^{e}}_{\frac{1}{d}}$ & $\vec{r}$ & $\underset{1}{N}$ & m & $\overrightarrow{4}$ & 노 & b & 牙 & 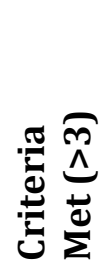 \\
\hline & & & & & & & & & & & & & & \\
\hline
\end{tabular}


IMPROVE HF SELF-CARE EDUCATION DELIVERY

\section{Appendix G}

Master List: Provider Identification

Template for Excel Spreadsheet

To Be Converted to Password Protected PDF

\begin{tabular}{|l|c|}
\hline Provider Name & Assigned Provider ID Code \\
\hline & \\
\hline
\end{tabular}


IMPROVE HF SELF-CARE EDUCATION DELIVERY

\section{Appendix H}

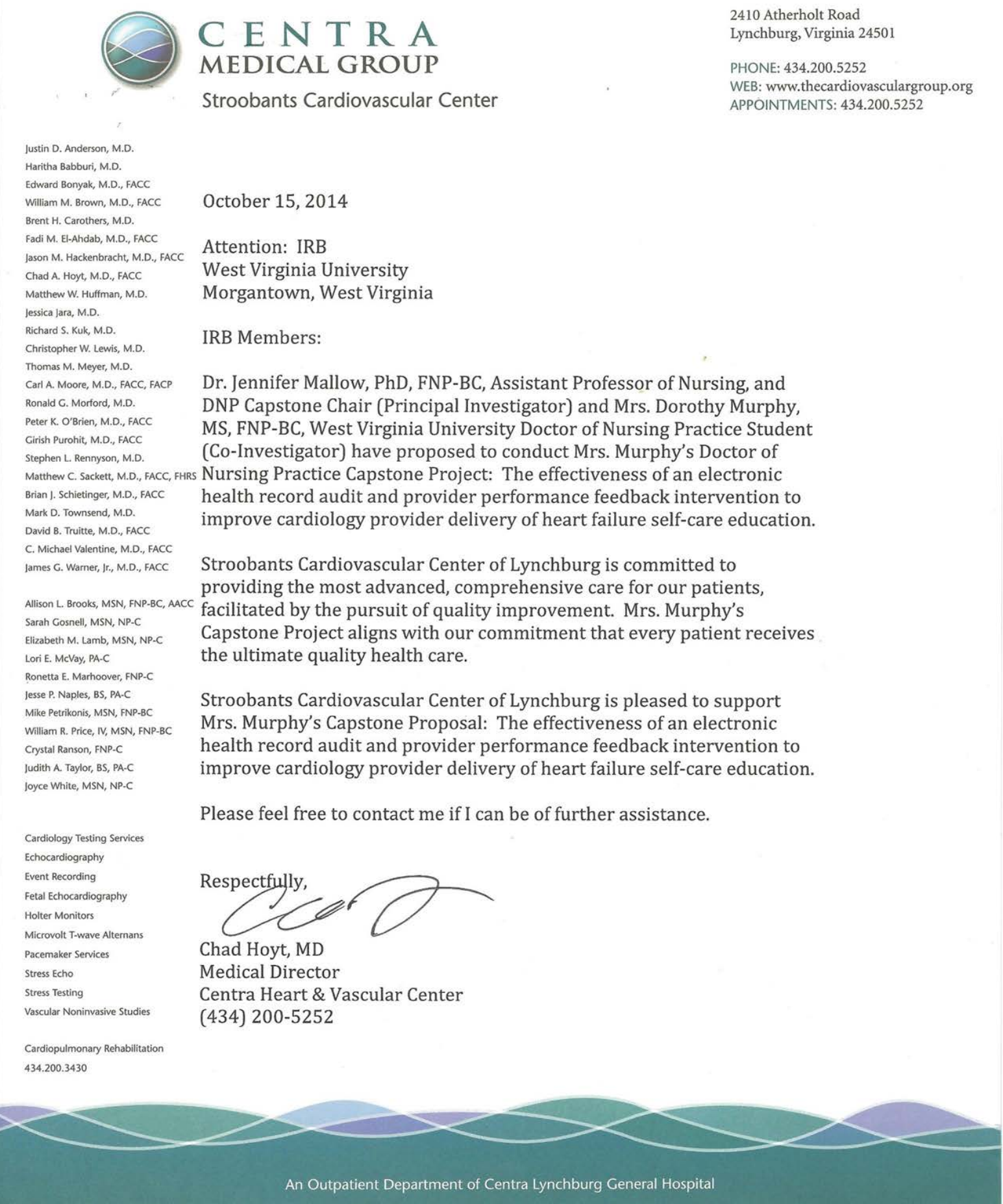




\section{Appendix I}

I d: 159270

From: admin

Recipients: dmurph10, jamallow

Channel: KC Notification Channel

Producer: Notification System

Type: FYI

Priority: Normal

Send Date: 2014-12-23T13:01: 11.000-05:00

Removal Date: none

Title: IRB Protocol Notice: Expedited Protocol 1410451583 Approved

Content:

I RB protocol number: 1410451583

Title: The effectiveness of an electronic health record audit and provider performance feedback intervention to improve cardiology providers' delivery of heart failure self-care education

\section{PI : Jennifer Mallow}

The West Virginia University I nstitutional Review Board approved the abovereferenced protocol on 23-Dec-2014. To access this protocol, click on the protocol number link provided. Your approval letter can be found in the History subsection of the Summary \& History section located on the Protocol Actions page. For more information, see the Viewing Correspondence quick reference guide. Any future protocol action requests can be completed through the WVU+kc system.

Questions related to Expedited protocols should be directed to Barbara White at 304.293.5971 304.293 .5971 or barb.white@mail.wvu.edu. 
IMPROVE HF SELF-CARE EDUCATION DELIVERY

\section{Appendix $\mathbf{J}$}

\section{E N T R A}

Lynchburg General Hospital
1901 Tate Springs Road

Lynchburg, Virginia 24501

PHONE: 434.200 .3000

WEB: CentraHealth.com

January 21, 2015

Jennifer Mallow, PhD, FNP-BC (jamallow@hsc.wvu.edu)

WVU School of Nursing

9620 HSC South

Morgantown, WV 26506

Dorothy Murphy, MS, FNP-BC (Dorothy.murphy@centrahealth.com)

Stroobant's Cardiovascular Center

2410 Atherholt Road

Lynchburg, VA 24501

Dear Dr. Mallow and Ms. Murphy:

At the meeting of the Centra Health Institutional Review Board on January 20, 2015 the research project and protocol CHIRB0270 The effectiveness of a chart audit and feedback intervention to improve cardiology provider delivery of heart failure self-care education request for approval described in the approval application (Reference 12/30/14) was approved.

We do require annual renewal or closure of the research project. This expiration date is JANUARY 20, 2016. A renewal or closure application will be due by JANUARY 5, 2016 to ensure this will be on the agenda for the January 19, 2016 IRB meeting.

If you have any questions, please consult the Centra Health IRB Policy and Procedure Manual http://foremplovees2.centrahealth.com/sites/IRB/WebPages/Instructions.aspx , or contact the IRB Secretary at 434-200-4513.

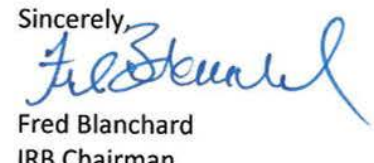

IRB Chairman

$\mathrm{FB} / \mathrm{bb}$ 